\title{
Global existence of classical solutions to the two-dimensional compressible Boussinesq equations in a square domain
}

\author{
Xucheng Huang ${ }^{1}$, Zhaoyang Shang ${ }^{1,2^{*}}$ (D) and Na Zhang ${ }^{3}$
}

"Correspondence:
shangzhaoyang@sjtu.edu.cn
'School of Finance, Shanghai Lixin
University of Accounting and
Finance, Shanghai 201209,
P.R. China
${ }^{2}$ School of Mathematical Sciences,
Shanghai Jiao Tong University,
Shanghai 200240, P.R. China
Full list of author information is
available at the end of the article

available at the end of the article

\begin{abstract}
In this paper, we consider the initial boundary value problem of two-dimensional isentropic compressible Boussinesq equations with constant viscosity and thermal diffusivity in a square domain. Based on the time-independent lower-order and time-dependent higher-order a priori estimates, we prove that the classical solution exists globally in time provided the initial mass $\left\|\rho_{0}\right\|_{L 1}$ of the fluid is small. Here, we have no small requirements for the initial velocity and temperature.
\end{abstract}

Keywords: Compressible Boussinesq equations; Small initial mass; Global classical solution

\section{Introduction}

In this paper, we consider the following two-dimensional isentropic compressible Boussinesq equations in the Eulerian coordinates:

$$
\left\{\begin{array}{l}
\rho_{t}+\operatorname{div}(\rho u)=0, \\
(\rho u)_{t}+\operatorname{div}(\rho u \otimes u)-\mu \Delta u+\nabla P=\rho \theta e_{2}, \\
\theta_{t}+(u \cdot \nabla) \theta-\kappa \Delta \theta=0,
\end{array}\right.
$$

where $\rho=\rho(x, t), u=\left(u_{1}, u_{2}\right)(x, t), \theta=\theta(x, t)$ are unknown functions denoting the density, velocity, and temperature of the fluid, respectively, $t \geq 0$ is time, $x \in \Omega$ is spatial coordinate. The pressure $P$ is given by

$$
P=A \rho^{\gamma},
$$

with constants $A>0, \gamma>0$. Both $\mu$ and $\kappa$ are nonnegative parameters denoting the viscosity and thermal diffusivity. The term $\rho \theta e_{2}$ with $e_{2}=(0,1)$ represents the buoyancy force.

(c) The Author(s) 2020. This article is licensed under a Creative Commons Attribution 4.0 International License, which permits use, sharing, adaptation, distribution and reproduction in any medium or format, as long as you give appropriate credit to the original author(s) and the source, provide a link to the Creative Commons licence, and indicate if changes were made. The images or other third party material in this article are included in the article's Creative Commons licence, unless indicated otherwise in a credit line to the material. If material is not included in the article's Creative Commons licence and your intended use is not permitted by statutory regulation or exceeds the permitted use, you will need to obtain permission directly from the copyright holder. To view a copy of this licence, visit http://creativecommons.org/licenses/by/4.0/. 
If $\rho$ and $u$ are regular enough, then (1.1) can be rewritten as follows:

$$
\left\{\begin{array}{l}
\rho_{t}+\operatorname{div}(\rho u)=0 \\
\rho\left(u_{t}+u \cdot \nabla u\right)-\mu \Delta u+\nabla P=\rho \theta e_{2} \\
\theta_{t}+(u \cdot \nabla) \theta-\kappa \Delta \theta=0 .
\end{array}\right.
$$

The Boussinesq equations have been widely used in atmospheric sciences, oceanic fluids, and as a model in many geophysical applications [25]. Usually, scholars study the following incompressible Boussinesq equations:

$$
\left\{\begin{array}{l}
u_{t}+u \cdot \nabla u-\mu \Delta u+\nabla P=\rho \theta e_{2} \\
\theta_{t}+(u \cdot \nabla) \theta-\kappa \Delta \theta=0 \\
\operatorname{div} u=0
\end{array}\right.
$$

and there is a huge amount of literature on the well-posedness theory of strong and classical solutions for the two-dimensional Boussinesq equations. First, let us review some well-posedness results of equations (1.3) in different cases.

(1) Constant viscosity and thermal diffusivity In 1980, Cannon and DiBenedetto [7] studied the Cauchy problem for the Boussinesq equations with full dissipation terms $(\mu>0, \kappa>0)$. They found a unique global-in-time weak solution. Moreover, they improved the regularity of the solution when the initial data are smooth. In 1997, Chae and Nam [10] considered inviscid flows $(\mu=\kappa=0)$ with external potential force and proved the local existence and uniqueness of smooth solutions. Furthermore, global existence of smooth solution is obtained in the case of zero external force with the initial data in a Sobolev space. For the Hölder continuous initial data, similar results can be found in [9]. In 2004, Sawada and Taniuchi [28] considered equation (1.3) with the term of acceleration of gravity and established the local existence and uniqueness of mild solutions in the $\mathrm{n}$-dimensional whole space with nondecaying initial data. In a two-dimensional space, the local solution can be extended globally in time without smallness of the initial data. In 2005, when $\kappa=0$, Hou and Li [17] proved the global well-posedness of the Cauchy problem of viscous Boussinesq equations for general initial data in $H^{m}, m \geq 3$. In 2006, Chae [8] proved the global-in-time regularity with either zero diffusivity or zero viscosity. He also proved that as diffusivity (viscosity) tends to zero, the solutions of fully viscous equations converge strongly to those of zero diffusion (viscosity) equations. We refer to [1] for the case of partial viscosity with the initial data in a Besov space. In 2011, Danchin and Paicu [13] studied the Boussinesq system with horizontal viscosity in only one equation and constructed global weak solutions and strong unique solutions with large initial data. In 2017, Qiu and Yao [27] considered the Cauchy problem of N-dimensional $(N \geq 2)$ incompressible density-dependent Boussinesq equations without dissipation terms. They established local well-posedness results under the framework of Besov spaces, where the initial density is bounded away from zero. When the initial data permit vacuum, in 2019, Zhong [46] considered the equations without dissipation term on the temperature equation $(\kappa=0)$ in the whole space $\mathbb{R}^{2}$. He showed that there exists 
a unique local strong solution provided the initial density and the initial temperature decay not too slow at infinity. In the same year, Zhong [45] proved the existence of global strong solution by using the same initial conditions as those in [46] and the coefficients are required $\mu>0, \kappa>0$.

(2) Variable viscosity and thermal diffusivity

When $\mu$ and $\kappa$ depend on the temperature and satisfy the following conditions:

$$
\inf _{\theta \in \mathbb{R}}\{\mu(\theta), \kappa(\theta)\}>0
$$

Lorca and Boldrini [24] proved the global existence of weak solutions and local existence of a strong solution to the initial boundary value problem in 1999. In 2011, Wang and Zhang [34] studied the Cauchy problem and proved the global existence of smooth solution, where they assumed that $\mu(\theta)$ and $\kappa(\theta)$ are some smooth functions satisfying

$$
C_{0}^{-1} \leq \mu(\theta) \leq C_{0}, \quad C_{0}^{-1} \leq \kappa(\theta) \leq C_{0}, \quad \theta \in \mathbb{R},
$$

for some positive constants. Global regularity for the initial boundary value problem was given by Sun and Zhang in [32]. In 2013, Li and Xu [20] considered the Cauchy problem of an inviscid Boussinesq system with temperature-dependent thermal diffusivity. They proved the global well-posedness of strong solutions for arbitrarily large initial data in Sobolev spaces. In 2015, Li, Pan, and Zhang [21] considered the initial boundary value problem of inviscid heat conductive Boussinesq equations over a bounded domain with smooth boundary. Under slip boundary condition of velocity and the homogeneous Dirichlet boundary condition for temperature, they showed that there exists a unique global smooth solution for $H^{3}$ initial data. Moreover, they also showed that the temperature converges exponentially to zero as time goes to infinity, and the velocity and vorticity are uniformly bounded in time. In 2016, Jiu and Liu [19] studied the global well-posedness of anisotropic nonlinear Boussinesq equations with horizontal temperature-dependent viscosity and vertical thermal diffusivity in the whole space. They built up a uniqueness criterion which together with the a priori estimates admits a unique global solution without any smallness assumptions. In 2018, Zhai and Chen [43] studied the global well-posedness issue for the Boussinesq system with the temperature-dependent viscosity in $\mathbb{R}^{n}$, they proved a global solution in $\mathbb{R}^{2}$ provided the initial temperature is exponentially small. Very recently, Ye [40] studied the nonhomogeneous density-temperature-dependent Boussinesq equations with zero diffusivity over bounded domains and obtained a blow-up criterion in terms of the gradient of viscosity for strong solutions with vacuum.

Global well-posedness with fractional partial dissipation can be found in recent works $[2,14,30,39,41,44]$. For local and global theories of solutions in a three-dimensional space, we refer to $[3,4,12,16,23,26,29,36,38]$ and the references cited therein.

Compared with incompressible Boussinesq equations, there are fewer results on the two-dimensional compressible Boussinesq equations (1.2). In 2009, Xu [37] considered the local existence for smooth solutions to the Cauchy problem of the equations with external force and extended the results in [10] to the compressible case, where $\mu=\kappa=0$ and the 
initial density is strictly positive. When the initial density need not be positive and may vanish in an open set, in 2014, Tang and Gao [33] considered the equations with $\kappa=0$ in $\mathbb{R}^{3}$ and proved the existence of unique local strong solutions for all initial data satisfying some compatibility conditions. However, the results concerning global existence of strong or classical solutions of compressible Boussinesq equations with the initial data permitting vacuum are very limited at present. In this paper, we consider the Dirichlet problem of (1.2) with the following initial boundary conditions:

$$
\begin{aligned}
& \left.(\rho, u, \theta)(x, t)\right|_{t=0}=\left(\rho_{0}(x), u_{0}(x), \theta_{0}(x)\right), \\
& \left.(u, \theta)(x, t)\right|_{\partial \Omega}=0,
\end{aligned}
$$

where the flow domain is taken to be the square

$$
\Omega=\left\{\left(x_{1}, x_{2}\right) \in \mathbb{R}^{2} \mid 0<x_{1}<1,0<x_{2}<1\right\} .
$$

We hope to establish the global existence of classical solutions for (1.2), (1.6)-(1.7) with constant viscosity and thermal diffusivity in a square domain.

Before stating the main results, we explain the notations and conventions used throughout this paper.

Notations:

- The standard Lebesgue and Sobolev spaces are defined as follows:

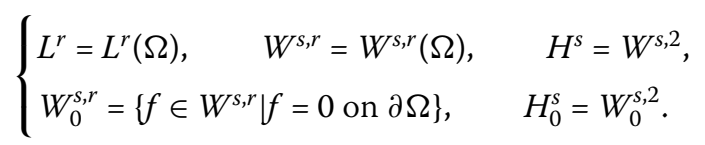

- $\dot{f}=f_{t}+u \cdot \nabla f$ denotes the material derivative of $f$.

- $\int f d x=\int_{\Omega} f d x$ and $\int_{0}^{T} \int f d x d t=\int_{0}^{T} \int_{\Omega} f d x d t$.

- The symbol $\nabla^{l}$ with an integer $l \geq 0$ stands for the usual any spatial derivatives of order $l$. We define

$$
\nabla^{k} f=\left\{\partial_{x}^{\alpha} f_{i}|| \alpha \mid=k, i=1,2\right\}, \quad f=\left(f_{1}, f_{2}\right)
$$

- Positive generic constants are denoted by $C$, which may change in different places.

Now, our main results in this paper can be stated as follows.

Theorem 1.1 Let $\Omega$ be a square domain in $\mathbb{R}^{2}$. For any given positive numbers $\bar{\rho}, M$, and $N$, suppose that the initial data $\left(\rho_{0}, u_{0}, \theta_{0}\right)$ satisfy

$$
\left\{\begin{array}{l}
0 \leq \inf \rho_{0} \leq \rho_{0} \leq \sup \rho_{0} \leq \bar{\rho} \\
\left\|\nabla u_{0}\right\|_{L^{2}} \leq \sqrt{M}, \quad\left\|\theta_{0}\right\|_{L^{2}} \leq \sqrt{N} \\
\rho_{0} \in H^{3}, \quad\left(u_{0}, \theta_{0}\right) \in H_{0}^{1} \cap H^{3}
\end{array}\right.
$$

and some necessary compatibility conditions. Then there exists a positive constant $\varepsilon_{0} d e-$ pending on $\bar{\rho}, M, N, \mu, \kappa$, and some other known constants but independent of $T$, such that 


$$
\left\|\rho_{0}\right\|_{L^{1}} \leq \varepsilon_{0}
$$

the initial boundary value problem (1.2), (1.6)-(1.7) admits a unique global classical solution $(\rho, u, \theta)$ in $\Omega \times(0,+\infty)$ satisfying, for any $0<T<+\infty$,

$$
\begin{aligned}
& 0 \leq \rho(x, t) \leq 2 \bar{\rho}+1, \quad x \in \Omega, t \geq 0, \\
& \left\{\begin{array}{l}
0 \leq \rho \in C\left([0, T] ; H^{3}\right), \quad \rho_{t} \in L^{\infty}\left(0, T ; H^{1}\right), \\
(u, \theta) \in C\left([0, T] ; H_{0}^{1} \cap H^{3}\right) \cap L^{2}\left(0, T ; H^{4}\right), \\
\left(u_{t}, \theta_{t}\right) \in L^{\infty}\left(0, T ; H_{0}^{1} \cap H^{2}\right), \quad \sqrt{\rho} u_{t} \in L^{\infty}\left(0, T ; L^{2}\right) .
\end{array}\right.
\end{aligned}
$$

Remark 1.1 Cho and Kim [11] considered the full Navier-Stokes equations for viscous polytropic fluids with nonnegative thermal conductivity in a three-dimensional space. They proved the existence of unique local strong solutions for all initial data satisfying some compatibility condition, where the initial density need not be positive and may vanish in an open set. Moreover, their results hold for both bounded and unbounded domains. In this paper, when $\Omega$ is a square domain in $\mathbb{R}^{2}$ and the initial data $\left(\rho_{0}, u_{0}, \theta_{0}\right)$ are smooth enough, it is not difficult to verify that the methods in [11] still work for proving the local existence of classical solutions to the initial boundary value problem (1.2), (1.6)-(1.7).

Remark 1.2 In Theorem 1.1, we give the global existence of classical solution to the initial boundary value problem (1.2), (1.6)-(1.7) provided the initial mass $\left\|\rho_{0}\right\|_{L^{1}}$ is small and $\mu>0, \kappa>0$. In fact, for the three-dimensional case, combining the methods in this paper and [35], similar results can also be proved in bounded and unbounded domains. However, the question whether the local classical solution given by $\mathrm{Xu}$ in [37] for the inviscid case and the local strong solution given by Tang and Gao in [33] for the partial dissipation case can be extended to global-in-time is still open at this moment.

Remark 1.3 In [22], the authors proved the global existence of classical solutions to the two-dimensional isentropic compressible Navier-Stokes equations when the initial mass $\left\|\rho_{0}\right\|_{L^{1}}$ of the fluid is small. In this paper, we hope to extend the results in [22] to the compressible Boussinesq equations. Compared with compressible Navier-Stokes equations, we need to deal with the interaction between the density, velocity, and temperature of the fluid, buoyancy force term $\rho \theta e_{2}$, for example. In [45], Zhong proved the existence of global strong solution to the incompressible density-dependent Boussinesq equations, where the divergence-free condition implies the upper bound of the density, plays an important role in his proof. However, for the compressible case, we do not need the divergence-free condition, and the key ingredient in our proof is to obtain a uniform a priori upper bound for the density function, by which the force term $\rho \theta e_{2}$ can be controlled in the lower-order a priori estimates of the velocity. On the one hand, similar to the procedure of [22], we are fortunate to obtain the following inequality under the smallness of $\left\|\rho_{0}\right\|_{L^{1}}=: m_{0}$, when the momentum equation is related to temperature:

$$
\sup _{0 \leq t \leq T}\left(\|\sqrt{\rho} u\|_{L^{2}}^{2}+\frac{2 A}{\gamma-1}\|\rho\|_{\gamma}^{\gamma}\right)+\int_{0}^{T} \mu\|\nabla u\|_{L^{2}}^{2} d s \leq m_{0}^{\frac{1}{4}}
$$


which lays the foundation of the time-independent lower-order a priori estimates. With (1.11) at hand, estimates on the first-order derivative of the temperature can be achieved by careful analysis. It should be pointed out that due to the strong coupling between velocity and temperature, we cannot take $\kappa=0$ as those in $[8,17,45]$.

On the other hand, in the process of estimating the first-order derivative of the velocity, we should control $\|\nabla u\|_{L^{p}}$ for some $p \geq 2$, which is equivalent to a second-order term. In this paper, we construct the following decomposition of the velocity $u=v+w$ to overcome this difficulty, where $v$ solves the elliptic system

$$
\left\{\begin{array}{l}
\mu \Delta v=\nabla P, \quad \text { in } \Omega, \\
\left.v\right|_{\partial \Omega}=0 .
\end{array}\right.
$$

Then, from momentum equation (1.2) and (1.12), we can see that $w$ satisfies

$$
\left\{\begin{array}{l}
\mu \Delta w=\rho \dot{u}+\rho \theta e_{2}, \quad \text { in } \Omega \\
\left.w\right|_{\partial \Omega}=0 .
\end{array}\right.
$$

Hence, by the standard elliptic estimate, we can see that $\|\nabla u\|_{L^{p}}$ can be controlled by the terms of $\|\sqrt{\rho} \dot{u}\|_{L^{2}},\|\nabla u\|_{L^{2}}$, and $\|\nabla \theta\|_{L^{2}}$, where the second-order term $\|\sqrt{\rho} \dot{u}\|_{L^{2}}$, in our estimates, can be absorbed by the left-hand side of the inequality, and the Poincaré inequality will be used frequently in our estimates. Then, applying the methods in [22, 42], we get the uniform bound for $\|\nabla u\|_{L^{2}}$. Similar to the proof in [22], together with the Zlotnik inequality, we get the uniform upper bound of the density. It is worth mentioning that this boundedness can be obtained only under the condition of the small initial mass $\left\|\rho_{0}\right\|_{L^{1}}$, we have no small requirements for the initial velocity and temperature. At last, compared with Navier-Stokes equations, we need some higher-order time-dependent estimates on the temperature, additionally. Then the higher-order regularity estimates for $(\rho, u, \theta)$ can be proved by standard methods after some modifications. Finally, after all the required a priori estimates are obtained, by using the continuity argument, we can extend the local classical solution to a global one.

The rest of the paper is organized as follows: In Sect. 2, we list some elementary inequalities which will be used in later analysis. In Sect. 3 and Sect. 4, we show the timeindependent lower-order and time-dependent higher-order a priori estimates, respectively, by which we prove that the classical solution exists globally in time provided the initial mass $\left\|\rho_{0}\right\|_{L^{1}}$ of the fluid is small.

\section{Preliminaries}

In this section, we recall some well-known inequalities, which will be used frequently throughout this paper. First, we give the following Sobolev-Poincaré inequalities, see in reference [6].

Lemma 2.1 There exists a positive constant $C$ depending only on $\Omega$ such that every function $f \in H^{1}(\Omega)$ satisfies, for $2<p<\infty$,

$$
\|f-\bar{f}\|_{L^{p}} \leq C p^{\frac{1}{2}}\|f-\bar{f}\|_{L^{2}}^{\frac{2}{\bar{p}}}\|\nabla f\|_{L^{2}}^{1-\frac{2}{\bar{p}}}, \quad\|f\|_{L^{p}} \leq C p^{\frac{1}{2}}\|f\|_{L^{2}}^{\frac{2}{p}}\|f\|_{H^{1}}^{1-\frac{2}{p}},
$$


where

$$
\bar{f}=\frac{1}{|\Omega|} \int_{\Omega} f d x
$$

Next, we give some regularity results for the following Lamé system with Dirichlet boundary condition (see [31]):

$$
\left\{\begin{array}{l}
\mathcal{L} U \triangleq \mu \Delta U+(\mu+\lambda) \nabla \operatorname{div} U=F, \quad x \in \Omega, \\
U=0, \quad x \in \partial \Omega
\end{array}\right.
$$

Suppose that $U \in H_{0}^{1}$ is a weak solution to the Lamé system, we could denote $U=\mathcal{L}^{-1} F$ due to the uniqueness of solution.

Lemma 2.2 Let $r \in(1,+\infty)$, then there exists some generic constant $C>0$ depending only on $\mu, \lambda, r$, and $\Omega$ such that

(1) If $F \in L^{r}$, then

$$
\|U\|_{W^{2, r}(\Omega)} \leq C\|F\|_{L^{r}(\Omega)}
$$

(2) If $F \in W^{-1, r}$, i.e., $F=\operatorname{divf}$ with $f=\left(f_{i, j}\right)_{2 \times 2}, f_{i, j} \in L^{r}$, then

$$
\|U\|_{W^{1, r}(\Omega)} \leq C\|f\|_{L^{r}(\Omega)} .
$$

(3) Moreover, for the endpoint case, iff $f_{i, j} \in L^{2} \cap L^{\infty}$, then $\nabla U \in B M O(\Omega)$ and

$$
\|U\|_{B M O(\Omega)} \leq C\left(\|f\|_{L^{2}(\Omega)}+\|f\|_{L^{\infty}(\Omega)}\right),
$$

where $B M O(\Omega)$ stands for the John-Nirenberg space of mean oscillation whose norm is defined by

$$
\|f\|_{B M O} \triangleq\|f\|_{L^{2}}+[f]_{B M O(\Omega)},
$$

with

$$
[f]_{B M O(\Omega)} \triangleq \sup _{x \in \Omega, r \in(0, d)} \frac{1}{\Omega_{r}(x)} \int_{\Omega_{r}(x)}\left|f(y)-f_{\Omega_{r}(x)}\right| d y,
$$

and

$$
f_{\Omega_{r}(x)}=\frac{1}{\Omega_{r}(x)} \int_{\Omega_{r}(x)} f(y) d y .
$$

In the following, we give two critical Sobolev inequalities of logarithmic type, which are originally due to Brezis and Gallouet [5] and Brezis and Wainger [15].

Lemma 2.3 Let $\Omega \in \mathbb{R}^{2}$ be a bounded Lipschitz domain and $f \in W^{1, q}$ with $q>2$, then it holds that

$$
\|f\|_{L^{\infty}(\Omega)} \leq C\left(1+\|f\|_{B M O(\Omega)} \ln \left(e+\|f\|_{W^{1, q}}\right)\right)
$$

with a constant $C$ depending only on $q$. 
Lemma 2.4 Let $\Omega \in \mathbb{R}^{2}$ be a smooth domain and $f \in L^{2}\left(s, t ; H_{0}^{1}\right) \cap L^{2}\left(s, t ; W^{1, q}\right)$, with some $q>2$ and $0 \leq s<t \leq \infty$. Then it holds that

$$
\|f\|_{L^{2}\left(s, t ; L^{\infty}\right)}^{2} \leq C\left(1+\|f\|_{L^{2}\left(s, t ; H^{1}\right)}^{2} \ln \left(e+\|f\|_{L^{2}\left(s, t ; W^{1, q}\right)}\right)\right)
$$

with a constant $C$ depending only on $q$.

Finally, we give a lemma arising from Zlotnik [47], which will be used to prove a uniform upper bound for density.

Lemma 2.5 Let $y \in W^{1,1}(0, T)$ satisfy the $O D E$ system

$$
y^{\prime}(t)=g(y)+b^{\prime}(t) \quad \text { on }[0, T], y(0)=y_{0},
$$

where $b \in W^{1,1}(0, T), g \in C(\mathbb{R})$, and $g(+\infty)=-\infty$. Assume that there are two constants $N_{0} \geq 0$ and $N_{1} \geq 0$ such that, for all $0 \leq t_{1} \leq t_{2} \leq T$,

$$
b\left(t_{2}\right)-b\left(t_{1}\right) \leq N_{0}+N_{1}\left(t_{2}-t_{1}\right) .
$$

Then

$$
y(t) \leq \max \left\{y_{0}, \xi^{\star}\right\}+N_{0}<+\infty \quad \text { on }[0, T]
$$

where $\xi^{\star} \in \mathbb{R}$ is a constant such that $g(\xi) \leq-N_{1}$ for $\xi \geq \xi^{\star}$.

\section{Time-independent lower-order estimates}

In this section, we establish some lower-order a priori estimates for the solutions of initial boundary value problem (1.2), (1.6)-(1.7). We assume that, for any $T>0,(\rho, u, \theta)$ is a classical solution of (1.2), (1.6)-(1.7) in the solution space (1.10) with the initial data satisfying (1.8). In the following Proposition 3.1, we show the time-independent lower-order estimates of the solutions.

Proposition 3.1 Assume that the initial data satisfy (1.8), and the local strong solution satisfies

$$
\begin{aligned}
& \sup _{0 \leq t \leq T}\|\theta\|_{L^{2}}^{2}+\int_{0}^{T} \kappa\|\nabla \theta\|_{L^{2}}^{2} d s \leq 2 K_{1}, \quad \sup _{0 \leq t \leq \sigma(T)}\|\nabla u\|_{L^{2}}^{2} \leq 2 K_{2}, \\
& \sup _{0 \leq t \leq T}\left(\sigma\|\nabla u\|_{L^{2}}^{2}\right) \leq 2 m_{0}^{\frac{1}{8}}, \quad 0 \leq \rho \leq 2 \bar{\rho}+1,(x, t) \in \Omega \times[0, T],
\end{aligned}
$$

where $\sigma(t) \triangleq \min \{1, t\}$. Then there exists $\varepsilon_{0}=\min \left\{\varepsilon_{1}^{*}, \varepsilon_{2}^{*}, \varepsilon_{3}^{*}, \varepsilon_{4}^{*}, \varepsilon_{5}^{*}\right\}$ depending on $\bar{\rho}, K_{1}, K_{2}$, $\mu, \kappa$, and some other known constants, but independent of $T$ such that

$$
\begin{aligned}
& \sup _{0 \leq t \leq T}\|\theta\|_{L^{2}}^{2}+\int_{0}^{T} \kappa\|\nabla \theta\|_{L^{2}}^{2} d s \leq \frac{3}{2} K_{1}, \quad \sup _{0 \leq t \leq \sigma(T)}\|\nabla u\|_{L^{2}}^{2} \leq \frac{3}{2} K_{2}, \\
& \sup _{0 \leq t \leq T}\left(\sigma\|\nabla u\|_{L^{2}}^{2}\right) \leq \frac{3}{2} m_{0}^{\frac{1}{8}}, \quad 0 \leq \rho \leq \frac{3}{2} \bar{\rho}+1,(x, t) \in \Omega \times[0, T],
\end{aligned}
$$

provided that $m_{0} \leq \varepsilon_{0}$ is suitably small. 
First, in order to prove Proposition 3.1, we give some basic equality and inequalities, which lay the foundation of time-independent lower-order a priori estimates.

Lemma 3.2 There exists a positive constant $C$ only depending on the initial data $\left(\rho_{0}, u_{0}, \theta_{0}\right)$ such that

$$
\begin{aligned}
& \sup _{0 \leq t \leq T}\|\rho\|_{L^{1}}=\left\|\rho_{0}\right\|_{L^{1}}=m_{0}, \\
& \sup _{0 \leq t \leq T}\left(\|\sqrt{\rho} u\|_{L^{2}}^{2}+\frac{2 A}{\gamma-1}\|\rho\|_{\gamma}^{\gamma}\right)+\int_{0}^{T} \mu\|\nabla u\|_{L^{2}}^{2} d s \leq m_{0}^{\frac{1}{4}}, \\
& \sup _{0 \leq t \leq T}\|\theta(t)\|_{L^{2}}^{2}+\int_{0}^{T} \kappa\|\nabla \theta(s)\|_{L^{2}}^{2} d s \leq \frac{3}{2} K_{1} .
\end{aligned}
$$

Moreover, we have

$$
\sup _{0 \leq t \leq T}\|\theta(t)\|_{H^{1}}^{2}+\int_{0}^{T} \kappa\|\nabla \theta(s)\|_{H^{1}}^{2} d s \leq C
$$

provided that there exists a constant $\varepsilon_{1}^{*}$ such that $m_{0} \leq \varepsilon_{1}^{*}$.

Proof Integrating (1.2) ${ }_{1}$ over $\Omega$, (3.5) can be easily obtained. Next, multiplying $(1.1)_{2}$ by $u$, integrating the resulting equation over $\Omega$, and using $(1.2)_{1}$, we have

$$
\begin{aligned}
& \frac{1}{2} \frac{d}{d t} \int \rho|u|^{2} d x+\mu \int|\nabla u|^{2} d x+(\mu+\lambda) \int|\operatorname{div} u|^{2} d x \\
& \quad=\int \rho \theta e_{2} u d x-\int \nabla P \cdot u d x .
\end{aligned}
$$

Then multiplying (1.2) ${ }_{1}$ by $A \rho^{r-1}$ yields that

$$
\frac{A}{r-1} \frac{d}{d t} \int \rho^{r} d x=\int u \cdot \nabla P d x
$$

Integrating (3.9) and (3.10) over time interval $(0, t)$ and adding the resulting equation together, we have

$$
\begin{aligned}
& \left(\frac{1}{2}\|\sqrt{\rho} u\|_{L^{2}}^{2}+\frac{1}{\gamma-1}\|\rho\|_{L^{\gamma}}^{\gamma}\right)+\int_{0}^{t} \mu\|\nabla u\|_{L^{2}}^{2} d s \\
& \quad \leq \frac{1}{2}\left\|\rho_{0}\right\|_{L^{2}}\left\|u_{0}\right\|_{L^{4}}^{2}+\frac{1}{\gamma-1}\left\|\rho_{0}\right\|_{L^{\gamma}}^{\gamma}+\int_{0}^{t}\|\rho\|_{L^{2}}\|\theta\|_{L^{4}}\|u\|_{L^{4}} d s \\
& \quad \leq C \bar{\rho}^{\frac{1}{2}}\left\|\nabla u_{0}\right\|_{L^{2}}^{2} m_{0}^{\frac{1}{2}}+C \bar{\rho}^{\gamma-1} m_{0}+\int_{0}^{t}\left(C \bar{\rho} m_{0}^{\frac{1}{2}}\|\nabla u\|_{L^{2}}^{2}+m_{0}^{\frac{1}{2}}\|\nabla \theta\|_{L^{2}}^{2}\right) d s \\
& \quad \leq \int_{0}^{t} \frac{\mu}{2}\|\nabla u\|_{L^{2}}^{2} d s+C \bar{\rho}^{-\frac{1}{2}} M m_{0}^{\frac{1}{2}}+C \bar{\rho}^{\gamma-1} m_{0}+C m_{0}^{\frac{1}{2}} K_{1} \\
& \quad \leq \int_{0}^{t} \frac{\mu}{2}\|\nabla u\|_{L^{2}}^{2} d s+m_{0}^{\frac{1}{4}},
\end{aligned}
$$


provided there exists a constant $\varepsilon_{1}, m_{0} \leq \varepsilon_{1}$, such that

$$
C \bar{\rho} m_{0}^{\frac{1}{2}} \leq \frac{\mu}{2}, \quad C \bar{\rho}^{\frac{1}{2}} M m_{0}^{\frac{1}{2}}+C \bar{\rho}^{\gamma-1} m_{0}+C m_{0}^{\frac{1}{2}} K_{1} \leq m_{0}^{\frac{1}{4}} .
$$

In the second inequality of (3.11) we have used the Poincaré inequality and Young's inequality. Hence, (3.6) is proved.

For the temperature equation, multiplying $(1.2)_{3}$ by $\theta$, integrating the result over $\Omega$, after integrating by parts, we have

$$
\begin{aligned}
\frac{1}{2} \frac{d}{d t}\|\theta\|_{L^{2}}^{2}+\kappa\|\nabla \theta\|_{L^{2}}^{2} & =\int \operatorname{div} u \theta^{2} d x \leq\|\nabla u\|_{L^{2}}\|\theta\|_{L^{4}}^{2} \leq\|\nabla u\|_{L^{2}}\|\theta\|_{L^{2}}\|\nabla \theta\|_{L^{2}} \\
& \leq \frac{\kappa}{2}\|\nabla \theta\|_{L^{2}}^{2}+C\|\nabla u\|_{L^{2}}^{2}\|\theta\|_{L^{2}}^{2} .
\end{aligned}
$$

Then, integrating the above equation over time interval $(0, t)$, we get

$$
\begin{aligned}
\|\theta\|_{L^{2}}^{2}+\int_{0}^{t} \kappa\|\nabla \theta\|_{L^{2}}^{2} d s & \leq\left\|\theta_{0}\right\|_{L^{2}}^{2}+C \int_{0}^{t}\|\nabla u\|_{L^{2}}^{2}\|\theta\|_{L^{2}}^{2} d s \leq N+C K_{1} \int_{0}^{t}\|\nabla u\|_{L^{2}}^{2} d s \\
& \leq K_{1}+C K_{1} m_{0}^{\frac{1}{4}}=K_{1}+\frac{1}{2} K_{1}=\frac{3}{2} K_{1}
\end{aligned}
$$

by which (3.7) is proved, provided there exists a constant $\varepsilon_{2}, m_{0} \leq \varepsilon_{2}$, such that

$$
N \leq K_{1}, \quad C m_{0}^{\frac{1}{4}} \leq \frac{1}{2}
$$

Next, applying the operator $\nabla$ to the both sides of temperature equation $(1.2)_{3}$ and multiplying the resulting equation by $\nabla \theta$, we get

$$
\begin{aligned}
\frac{1}{2} \frac{d}{d t}\|\nabla \theta(t)\|_{L^{2}}^{2}+\kappa\left\|\nabla^{2} \theta\right\|_{L^{2}}^{2} & =-\int \nabla(u \cdot \nabla \theta) \cdot \nabla \theta d x \leq\|\nabla u\|_{L^{2}}\|\nabla \theta\|_{L^{4}}^{2} \\
& \leq C\|\nabla u\|_{L^{2}}\|\nabla \theta\|_{L^{2}}\left\|\nabla^{2} \theta\right\|_{L^{2}} \\
& \leq \frac{\kappa}{2}\left\|\nabla^{2} \theta\right\|_{L^{2}}^{2}+C\|\nabla u\|_{L^{2}}^{2}\|\nabla \theta\|_{L^{2}}^{2} .
\end{aligned}
$$

Integrating (3.14) over the time interval $(0, t)$ and using Gronwall's inequality, one can get (3.8). Hence, Lemma 3.2 is proved provided that there exists a constant $\varepsilon_{1}^{*}, \varepsilon_{1}^{*} \leq \min \left\{\varepsilon_{1}, \varepsilon_{2}\right\}$ such that $m_{0} \leq \varepsilon_{1}^{*}$.

Next, in the following lemma, we prove the uniform upper bound of $\|\nabla u\|_{L^{2}}$.

Lemma 3.3 Let $(\rho, u, \theta)$ be a classical solution of (1.2), (1.6)-(1.7) on $\Omega \times(0, T]$, if the assumption of Proposition 3.1 holds, then

$$
\begin{aligned}
& \sup _{0 \leq t \leq \sigma(T)}\|\nabla u\|_{L^{2}}^{2}+\int_{0}^{\sigma(T)}\|\sqrt{\rho} \dot{u}\|_{L^{2}}^{2} d s \leq \frac{3}{2} K, \\
& \sup _{i-1 \leq t \leq i+1}\left(\sigma\|\nabla u\|_{L^{2}}^{2}\right)+\int_{i-1}^{i+1} \sigma_{i}\|\sqrt{\rho} \dot{u}\|_{L^{2}}^{2} d s \leq \frac{3}{2} m_{0}^{\frac{1}{8}},
\end{aligned}
$$


provided there exists constant $\varepsilon_{2}^{*}$ such that $m_{0} \leq \min \left\{\varepsilon_{1}^{*}, \varepsilon_{2}^{*}\right\}$, where $\sigma_{i}(t) \triangleq \sigma(t+1-i)$, $1 \leq i \leq[T]-1, t \in(i-1, i+1]$.

Proof From $(1.2)_{2}$, we get

$$
\rho \dot{u}+\nabla P=\mu \Delta u+\rho \theta e_{2},
$$

multiplying (3.17) by $\eta \dot{u}, \eta=\eta(t) \geq 0$ is a piecewise smooth function. Integrating the resulting equation over $\Omega$ yields

$$
\begin{aligned}
\frac{1}{2} \frac{d}{d t} \int \mu \eta|\nabla u|^{2} d x+\int \eta \rho|\dot{u}|^{2} d x \\
=\frac{d}{d t} \int \eta P \operatorname{div} u d x-\int \eta^{\prime} P \operatorname{div} u d x-\int \eta\left(P_{t}+\operatorname{div}(P u)\right) \operatorname{div} u d x \\
\quad+\int \eta P \nabla u: \nabla u^{\top} d x-\mu \int \eta \nabla u:(\nabla u \nabla u) d x+\frac{1}{2} \mu \int \eta \operatorname{div} u|\nabla u|^{2} d x \\
\quad+\frac{1}{2} \mu \int \eta^{\prime}|\nabla u|^{2} d x+\int \rho \theta e_{2} \eta \dot{u} d x \\
:=\frac{d}{d t} \int \eta P \operatorname{div} u d x+\sum_{i=1}^{7} I_{i} .
\end{aligned}
$$

Now, we estimate $I_{i}, i=1,2,3, \ldots, 7$, one by one:

$$
\begin{aligned}
& I_{1} \leq\left|\eta^{\prime}\right|\|P\|_{L^{2}}\|\nabla u\|_{L^{2}} \leq\left|\eta^{\prime}\right|\|P\|_{L^{2}}^{2}+\left|\eta^{\prime}\right|\|\nabla u\|_{L^{2}}^{2} \leq\left|\eta^{\prime}\right| \bar{\rho}^{2 \gamma-1} m_{0}+\left|\eta^{\prime}\right|\|\nabla u\|_{L^{2}}^{2}, \\
& I_{2}+I_{3} \leq C \eta\|\nabla u\|_{L^{2}}^{2},
\end{aligned}
$$

where we have used the identities $P_{t}+\operatorname{div}(P u)=(1-\gamma) \rho^{\gamma} \operatorname{div} u$.

The terms $I_{4}, I_{5}$ can be estimated as follows:

$$
\begin{aligned}
I_{4}+I_{5} & \leq C \eta\|\nabla u\|_{L^{3}}^{3} \leq C \eta\left(\|\nabla v\|_{L^{3}}^{2}+\|\nabla w\|_{L^{3}}^{3}\right) \\
& \leq C \eta\left(\|P\|_{L^{3}}^{3}+\|\nabla w\|_{L^{2}}^{2}\left\|\nabla^{2} w\right\|_{L^{2}}\right) \\
& \leq C \eta\left(\bar{\rho}^{3 \gamma-1} m_{0}+\left(\|\nabla u\|_{L^{2}}^{2}+\|\nabla v\|_{L^{2}}^{2}\right)\left\|\nabla^{2} w\right\|_{L^{2}}\right) \\
& \leq C \eta\left(\bar{\rho}^{3 \gamma-1} m_{0}+\left(\|\nabla u\|_{L^{2}}^{2}+\|P\|_{L^{2}}^{2}\right)\left\|\nabla^{2} w\right\|_{L^{2}}\right) \\
& \leq C \eta\left(\bar{\rho}^{3 \gamma-1} m_{0}+\left(\|\nabla u\|_{L^{2}}^{2}+\bar{\rho}^{2 \gamma-1} m_{0}\right)\left(\|\sqrt{\rho} \dot{u}\|_{L^{2}}+\left\|\rho \theta e_{2}\right\|_{L^{2}}\right)\right) \\
& \leq C \eta\left(m_{0}+\left(\|\nabla u\|_{L^{2}}^{2}+m_{0}\right)\left(\|\sqrt{\rho} \dot{u}\|_{L^{2}}+\|\rho\|_{L^{4}}\|\theta\|_{L^{4}}\right)\right) \\
& \leq C \eta\left(m_{0}+\left(\|\nabla u\|_{L^{2}}^{2}+m_{0}\right)\left(\|\sqrt{\rho} \dot{u}\|_{L^{2}}+m_{0}^{\frac{1}{4}}\|\nabla \theta\|_{L^{2}}\right)\right) \\
& \leq C \eta\left(m_{0}+m_{0}^{2}\right)+\frac{1}{4} \eta\|\sqrt{\rho} \dot{u}\|_{L^{2}}^{2}+C \eta\|\nabla u\|_{L^{2}}^{4}+\eta m_{0}^{\frac{1}{2}}\|\nabla \theta\|_{L^{2}}^{2},
\end{aligned}
$$

where in the last inequality we have used the Poincaré inequality.

At last, from terms $I_{6}, I_{7}$, we get

$$
I_{6}+I_{7} \leq C\left|\eta^{\prime}\right|\|\nabla u\|_{L^{2}}^{2}+\frac{1}{4} \eta\|\sqrt{\rho} \dot{u}\|_{L^{2}}^{2}+C \eta \int \rho \theta^{2} d x
$$


Huang et al. Journal of Inequalities and Applications

(2020) 2020:232

Page 12 of 32

$$
\begin{aligned}
& \leq C\left|\eta^{\prime}\right|\|\nabla u\|_{L^{2}}^{2}+\frac{1}{4} \eta\|\sqrt{\rho} \dot{u}\|_{L^{2}}^{2}+C \eta\|\rho\|_{L^{2}}\|\theta\|_{L^{4}}^{2} \\
& \leq C\left|\eta^{\prime}\right|\|\nabla u\|_{L^{2}}^{2}+\frac{1}{4} \eta\|\sqrt{\rho} \dot{u}\|_{L^{2}}^{2}+C \eta m_{0}^{\frac{1}{2}}\|\nabla \theta\|_{L^{2}}^{2} .
\end{aligned}
$$

Then, inserting (3.19)-(3.22) into (3.18), we have

$$
\begin{aligned}
& \frac{1}{2} \frac{d}{d t} \int \mu \eta|\nabla u|^{2} d x+\frac{1}{2} \int \eta \rho|\dot{u}|^{2} d x \\
& \leq \frac{d}{d t} \int \eta P \operatorname{div} u d x+C\left|\eta^{\prime}\right| m_{0}+C \eta\left(m_{0}+m_{0}^{2}\right) \\
& \quad+C\left(\left|\eta^{\prime}\right|+\eta\right)\|\nabla u\|_{L^{2}}^{2}+C \eta\left(\|\nabla u\|_{L^{2}}^{4}+m_{0}^{\frac{1}{2}}\|\nabla \theta\|_{L^{2}}^{2}\right) .
\end{aligned}
$$

In order to prove (3.15), taking $\eta=1$ and integrating (3.23) over $(0, t)$, for $0<t \leq \sigma(T)$, we get

$$
\begin{aligned}
& \int \mu|\nabla u|^{2} d x+\int_{0}^{\sigma(T)} \int \rho|\dot{u}|^{2} d x d s \\
& \leq \mu\left\|\nabla u_{0}\right\|_{L^{2}}^{2}+\|P\|_{L^{2}}\|\nabla u\|_{L^{2}}+\left\|P_{0}\right\|_{L^{2}}\left\|\nabla u_{0}\right\|_{L^{2}}+C\left(m_{0}+m_{0}^{2}\right)+C m_{0}^{\frac{1}{4}} \\
& \quad+C\left(K_{2} m_{0}^{\frac{1}{4}}+K_{1} m_{0}^{\frac{1}{2}}\right) \\
& \quad \leq \mu M+C\left(\sqrt{K_{2}} m_{0}^{\frac{1}{2}}+\sqrt{M} m_{0}^{\frac{1}{2}}+m_{0}^{\frac{1}{2}}+m_{0}+m_{0}^{2}+m_{0}^{\frac{1}{4}}+K_{2} m_{0}^{\frac{1}{4}}+K_{1} m_{0}^{\frac{1}{2}}\right),
\end{aligned}
$$

where we have used (3.1) and (3.6). Then we have

$$
\sup _{0 \leq t \leq \sigma(T)} \int \mu|\nabla u|^{2} d x+\int_{0}^{\sigma(T)} \int \rho|\dot{u}|^{2} d x d s \leq K_{2}+\frac{1}{2} K_{2}=\frac{3}{2} K_{2}
$$

provided there exists a constant $\varepsilon_{3}, m_{0} \leq \varepsilon_{3}$, such that

$$
\mu M \leq K_{2}, \quad C\left(\sqrt{K_{2}} m_{0}^{\frac{1}{2}}+\sqrt{M} m_{0}^{\frac{1}{2}}+m_{0}^{\frac{1}{2}}+m_{0}+m_{0}^{2}+m_{0}^{\frac{1}{4}}+K_{2} m_{0}^{\frac{1}{4}}+K_{1} m_{0}^{\frac{1}{2}}\right) \leq \frac{1}{2} K_{2} .
$$

In order to prove (3.16), taking $\eta=\sigma_{i}$ in (3.23), integrating (3.23) over $(i-1, t)$, we get

$$
\begin{aligned}
& \sigma_{i} \int \mu|\nabla u|^{2} d x+\int_{i-1}^{t} \int \sigma_{i} \rho|\dot{u}|^{2} d x d s \\
& \leq \sigma_{i}\|P\|_{L^{2}}\|\nabla u\|_{L^{2}}+C\left(m_{0}+m_{0}^{2}+m_{0}^{\frac{1}{4}}\right)+C \int_{i-1}^{t} \sigma_{i}\|\nabla u\|_{L^{2}}^{4} d s \\
& \quad+C m_{0}^{\frac{1}{2}} \int_{i-1}^{t} \sigma_{i}\|\nabla \theta\|_{L^{2}}^{2} d s \\
& \leq \frac{1}{2} \sigma_{i} \mu\|\nabla u\|_{L^{2}}^{2}+C\left(m_{0}+m_{0}^{2}+m_{0}^{\frac{1}{4}}\right)+C \sup _{i-1 \leq t \leq i+1} \sigma_{i}\|\nabla u\|_{L^{2}}^{2} \int_{i-1}^{t}\|\nabla u\|_{L^{2}}^{2} d s \\
& \quad+C m_{0}^{\frac{1}{2}} \int_{0}^{T}\|\nabla \theta\|_{L^{2}}^{2} d s \\
& \leq \frac{1}{2} \sigma_{i} \mu\|\nabla u\|_{L^{2}}^{2}+C\left(m_{0}+m_{0}^{2}+m_{0}^{\frac{1}{4}}+m_{0}^{\frac{1}{2}} K_{1}\right)+C \sup _{i-1 \leq t \leq i+1} \sigma_{i}\|\nabla u\|_{L^{2}}^{2} m_{0}^{\frac{1}{4}}
\end{aligned}
$$




$$
\leq \frac{1}{2} \sigma_{i} \mu\|\nabla u\|_{L^{2}}^{2}+C\left(m_{0}+m_{0}^{2}+m_{0}^{\frac{1}{4}}+m_{0}^{\frac{3}{8}}+m_{0}^{\frac{1}{2}} K_{1}\right) .
$$

Then we have

$$
\sup _{i-1 \leq t \leq i+1} \sigma_{i} \int \mu|\nabla u|^{2} d x+\int_{i-1}^{i+1} \int \sigma_{i} \rho|\dot{u}|^{2} d x d s \leq \frac{3}{2} m_{0}^{\frac{1}{8}},
$$

provided there exists a constant $\varepsilon_{4}, m_{0} \leq \varepsilon_{4}$, such that

$$
C\left(m_{0}+m_{0}^{2}+m_{0}^{\frac{1}{4}}+m_{0}^{\frac{3}{8}}+m_{0}^{\frac{1}{2}} K_{1}\right) \leq \frac{3}{2} m_{0}^{\frac{1}{8}} .
$$

Hence, if we take $\varepsilon_{2}^{*}=\min \left\{\varepsilon_{3}, \varepsilon_{4}\right\}, m_{0} \leq \min \left\{\varepsilon_{1}^{*}, \varepsilon_{2}^{*}\right\}$, then Lemma 3.3 is proved.

In the following Lemma 3.4, we give the bound for $\int_{t_{1}}^{t_{2}} \sigma^{2}\|\nabla \dot{u}\|_{L^{2}}^{2} d s$, which will be used to prove the uniform upper bound of $\rho$. It should be noted that the constants $C$ on the right-hand side of (3.28) and (3.29) are independent of time.

Lemma 3.4 Let $(\rho, u, \theta)$ be a classical solution of (1.2), (1.6)-(1.7) on $\Omega \times(0, T]$, if the assumption of Proposition 3.1 holds, then

$$
\begin{aligned}
& \sup _{0 \leq t \leq T}\left(\sigma^{2}\|\sqrt{\rho} \dot{u}\|_{L^{2}}^{2}\right) \leq C m_{0}^{\frac{1}{8}}, \\
& \int_{t_{1}}^{t_{2}} \sigma^{2}\|\nabla \dot{u}\|_{L^{2}}^{2} d s \leq C m_{0}\left(t_{2}-t_{1}\right)+C m_{0}^{\frac{1}{8}}
\end{aligned}
$$

for any $t_{1}, t_{2} \in(0, T]$, provided $m_{0} \leq \min \left\{\varepsilon_{1}^{*}, \varepsilon_{2}^{*}\right\}$.

Proof Operating $\eta \dot{u}^{j}\left(\partial_{t}+\operatorname{div}(u \cdot)\right)$ to $(1.2)_{2}^{j}$, summing with respect to $j$, and integrating the resulting equation over $\Omega$, we obtain

$$
\begin{aligned}
& \frac{d}{d t} \int \eta \rho|\dot{u}|^{2} d x-\eta^{\prime} \int \rho|\dot{u}|^{2} d x \\
& =-2 \eta \int \dot{u}^{j}\left(\partial_{j} P_{t}+\operatorname{div}\left(u \partial_{j} P\right)\right) d x+2 \mu \eta \int \dot{u}^{j}\left(\partial_{t} \Delta u^{j}+\operatorname{div}\left(u \Delta u^{j}\right)\right) d x \\
& \quad+2 \eta \int \dot{u}^{j}\left(\partial_{t}\left(\rho \theta e_{2}\right)^{j}+\operatorname{div}\left(u\left(\rho \theta e_{2}\right)^{j}\right)\right) d x \\
& \quad:=\sum_{i=1}^{3} J_{i} .
\end{aligned}
$$

It follows from integration by parts and using equation $(1.2)_{1}$ that

$$
\begin{aligned}
J_{1} & =-2 \eta \int \dot{u}^{j}\left(\partial_{j} P_{t}+\partial_{j} \operatorname{div}(u P)-\operatorname{div}\left(P \partial_{j} u\right)\right) d x \\
& =2 \eta \int \operatorname{div} \dot{u}\left(P_{t}+\operatorname{div}(u P)\right) d x-2 \eta \int\left(P \partial_{j} u\right) \cdot \nabla \dot{u}^{j} d x \\
& \leq C \eta \int \rho^{\gamma}|\operatorname{div} \dot{u}||\operatorname{div} u|^{2} d x+C \eta \int \rho^{\gamma}|\nabla u \| \nabla \dot{u}| d x \\
& \leq \frac{\mu \eta}{4}\|\nabla \dot{u}\|_{L^{2}}^{2}+C \eta\|\nabla u\|_{L^{2}}^{2},
\end{aligned}
$$




$$
\begin{aligned}
J_{2} & =2 \mu \eta \int \dot{u}^{j}\left[\Delta \dot{u}^{j}+\partial_{i}\left(\operatorname{div} u \partial_{i} u^{j}-\partial_{i} u \cdot \nabla u^{j}\right)-\operatorname{div}\left(\partial_{i} u \partial_{i} u^{j}\right)\right] d x \\
& =-2 \mu \eta \int\left|\nabla \dot{u}^{j}\right|^{2} d x-2 \mu \eta \int \partial_{i} \dot{u}^{j}\left(\operatorname{div} u \partial_{i} u^{j}-\partial_{i} u \cdot \nabla u^{j}\right) d x+2 \mu \eta \int \nabla \dot{u}^{j} \partial_{i} u \partial_{i} u^{j} d x \\
& \leq-\mu \eta\|\nabla \dot{u}\|_{L^{2}}^{2}+C \eta\|\nabla u\|_{L^{4}}^{4} \\
& \leq-\mu \eta\|\nabla \dot{u}\|_{L^{2}}^{2}+C \eta\left(m_{0}+\left(\|\nabla u\|_{L^{2}}^{2}+m_{0}\right)\left(\|\sqrt{\rho} \dot{u}\|_{L^{2}}^{2}+m_{0}^{\frac{1}{2}}\|\nabla \theta\|_{L^{2}}^{2}\right)\right)
\end{aligned}
$$

where $\|\nabla u\|_{L^{4}}^{4}$ can be estimated as follows:

$$
\begin{aligned}
\|\nabla u\|_{L^{4}}^{4} & \leq\|\nabla v\|_{L^{4}}^{4}+\|\nabla w\|_{L^{4}}^{4} \leq\|P\|_{L^{4}}^{4}+\|\nabla w\|_{L^{2}}^{2}\left\|\nabla^{2} w\right\|_{L^{2}}^{2} \\
& \leq C\left(\bar{\rho}^{4 \gamma-1} m_{0}+\left(\|\nabla u\|_{L^{2}}^{2}+\|\nabla v\|_{L^{2}}^{2}\right)\left\|\nabla^{2} w\right\|_{L^{2}}^{2}\right) \\
& \leq C\left(\bar{\rho}^{4 \gamma-1} m_{0}+\left(\|\nabla u\|_{L^{2}}^{2}+\|P\|_{L^{2}}^{2}\right)\left\|\nabla^{2} w\right\|_{L^{2}}^{2}\right) \\
& \leq C\left(m_{0}+\left(\|\nabla u\|_{L^{2}}^{2}+m_{0}\right)\left(\|\sqrt{\rho} \dot{u}\|_{L^{2}}^{2}+\left\|\rho \theta e_{2}\right\|_{L^{2}}^{2}\right)\right) \\
& \leq C\left(m_{0}+\left(\|\nabla u\|_{L^{2}}^{2}+m_{0}\right)\left(\|\sqrt{\rho} \dot{u}\|_{L^{2}}^{2}+\|\rho\|_{L^{4}}^{2}\|\theta\|_{L^{4}}^{2}\right)\right) \\
& \leq C\left(m_{0}+\left(\|\nabla u\|_{L^{2}}^{2}+m_{0}\right)\left(\|\sqrt{\rho} \dot{u}\|_{L^{2}}^{2}+m_{0}^{\frac{1}{2}}\|\nabla \theta\|_{L^{2}}^{2}\right)\right) .
\end{aligned}
$$

Similarly, we get

$$
\begin{aligned}
J_{3} & =2 \eta \int \dot{u}^{j}\left(\partial_{t}\left(\rho \theta e_{2}\right)^{j}+\operatorname{div}\left(u\left(\rho \theta e_{2}\right)^{j}\right)\right) d x=2 \eta \int \dot{u}^{j}\left(\rho \theta_{t} e_{2}^{j}+\rho u \nabla \theta e_{2}^{j}\right) d x \\
& \leq C \eta\left(\|\dot{u}\|_{L^{4}}\|\rho\|_{L^{4}}\left\|\theta_{t}\right\|_{L^{2}}+\|\dot{u}\|_{L^{4}}\|\rho\|_{L^{4}}\|u\|_{L^{4}}\|\nabla \theta\|_{L^{4}}\right) \\
& \leq C \eta\left(\|\nabla \dot{u}\|_{L^{2}} \bar{\rho}^{\frac{3}{4}} m_{0}^{\frac{1}{4}}\left(\|u \cdot \nabla \theta\|_{L^{2}}+\left\|\nabla^{2} \theta\right\|_{L^{2}}\right)+\|\nabla \dot{u}\|_{L^{2}} \bar{\rho}^{\frac{3}{4}} m_{0}^{\frac{1}{4}}\|\nabla u\|_{L^{2}}\|\nabla \theta\|_{L^{4}}\right) \\
& \leq C \eta\left(\|\nabla \dot{u}\|_{L^{2}} m_{0}^{\frac{1}{4}}\left(\|\nabla u\|_{L^{2}}\|\nabla \theta\|_{L^{4}}+\left\|\nabla^{2} \theta\right\|_{L^{2}}\right)+\|\nabla \dot{u}\|_{L^{2}} m_{0}^{\frac{1}{4}}\|\nabla u\|_{L^{2}}\|\nabla \theta\|_{L^{4}}\right) \\
& \leq \frac{\mu \eta}{4}\|\nabla \dot{u}\|_{L^{2}}^{2}+C \eta m_{0}^{\frac{1}{2}}\|\nabla u\|_{L^{2}}^{2}\|\nabla \theta\|_{L^{2}}\left\|\nabla^{2} \theta\right\|_{L^{2}},
\end{aligned}
$$

where we have used $(1.2)_{1},(1.2)_{3}$, and the Poincaré inequality. Then, substituting the estimates $J_{1}, J_{2}, J_{3}$ and (3.33) into (3.30), we arrive at

$$
\begin{aligned}
& \frac{d}{d t} \int \eta \rho|\dot{u}|^{2} d x+\frac{\mu \eta}{2}\|\nabla \dot{u}\|_{L^{2}}^{2} \\
& \quad \leq\left|\eta^{\prime}\right| \int \rho|\dot{u}|^{2} d x+C \eta\|\nabla u\|_{L^{2}}^{2}+C \eta\left(\|\nabla u\|_{L^{2}}^{2}+m_{0}\right)\left(\|\sqrt{\rho} \dot{u}\|_{L^{2}}^{2}+m_{0}^{\frac{1}{2}}\|\nabla \theta\|_{L^{2}}^{2}\right) \\
& \quad+C \eta m_{0}^{\frac{1}{2}}\|\nabla u\|_{L^{2}}^{2}\|\nabla \theta\|_{L^{2}}\left\|\nabla^{2} \theta\right\|_{L^{2}}+C \eta m_{0} .
\end{aligned}
$$

In order to prove (3.28), taking $\eta=\sigma_{i}^{2}$ in (3.35), integrating (3.35) over $(i-1, t)$, and taking (3.16) into consideration, we get

$$
\begin{aligned}
& \sigma_{i}^{2}\|\sqrt{\rho} \dot{u}\|_{L^{2}}^{2}+\int_{i-1}^{t} \mu \sigma_{i}^{2}\|\nabla \dot{u}\|_{L^{2}}^{2} d s \\
& \quad \leq \int_{i-1}^{t} \sigma_{i} \sigma_{i}^{\prime}\|\sqrt{\rho} \dot{u}\|_{L^{2}}^{2} d s+C \int_{i-1}^{t} \sigma_{i}^{2}\left(\|\nabla u\|_{L^{2}}^{2}+m_{0}\right)\left(\|\sqrt{\rho} \dot{u}\|_{L^{2}}^{2}+m_{0}^{\frac{1}{2}}\|\nabla \theta\|_{L^{2}}^{2}\right) d s
\end{aligned}
$$




$$
\begin{aligned}
& +C \int_{i-1}^{t} \sigma_{i}^{2} m_{0}^{\frac{1}{2}}\|\nabla u\|_{L^{2}}^{2}\|\nabla \theta\|_{L^{2}}\left\|\nabla^{2} \theta\right\|_{L^{2}} d s+C\left(m_{0}^{\frac{1}{4}}+m_{0}\right) \\
\leq & C m_{0}^{\frac{1}{8}}+C\left(\sup _{i-1 \leq t \leq i+1} \sigma_{i}\|\nabla u\|_{L^{2}}^{2}+m_{0}\right) \int_{i-1}^{t} \sigma_{i}\left(\|\sqrt{\rho} \dot{u}\|_{L^{2}}^{2}+m_{0}^{\frac{1}{2}}\|\nabla \theta\|_{L^{2}}^{2}\right) d s \\
& +C m_{0}^{\frac{1}{2}} \sup _{i-1 \leq t \leq i+1} \sigma_{i}\|\nabla u\|_{L^{2}}^{2}\|\nabla \theta\|_{L^{2}} \int_{i-1}^{t} \sigma_{i}\left\|\nabla^{2} \theta\right\|_{L^{2}} d s+C\left(m_{0}^{\frac{1}{4}}+m_{0}\right) \\
\leq & C m_{0}^{\frac{1}{8}}+C\left(m_{0}^{\frac{1}{8}}+m_{0}\right)\left(m_{0}^{\frac{1}{8}}+m_{0}^{\frac{1}{2}}\right)+C\left(m_{0}^{\frac{1}{4}}+m_{0}^{\frac{5}{8}}+m_{0}\right) \\
\leq & C m_{0}^{\frac{1}{8}},
\end{aligned}
$$

which proves (3.28).

Furthermore, from (3.35), we can see that if we take $\eta=\sigma^{2}$, then integrate (3.35) over $\left(t_{1}, t_{2}\right) \in[0, T]$, we have

$$
\begin{aligned}
\sigma^{2} \| & \sqrt{\rho} \dot{u}\left(t_{2}\right)\left\|_{L^{2}}^{2}+\int_{t_{1}}^{t_{2}} \mu \sigma^{2}\right\| \nabla \dot{u} \|_{L^{2}}^{2} d s \\
\leq & \sigma^{2}\left\|\sqrt{\rho} \dot{u}\left(t_{1}\right)\right\|_{L^{2}}^{2}+\int_{t_{1}}^{t_{2}} \sigma \sigma^{\prime}\|\sqrt{\rho} \dot{u}\|_{L^{2}}^{2} d s \\
& +C \int_{t_{1}}^{t_{2}} \sigma^{2}\left(\|\nabla u\|_{L^{2}}^{2}+m_{0}\right)\left(\|\sqrt{\rho} \dot{u}\|_{L^{2}}^{2}+m_{0}^{\frac{1}{2}}\|\nabla \theta\|_{L^{2}}^{2}\right) d s \\
& +C \int_{i-1}^{t} \sigma^{2} m_{0}^{\frac{1}{2}}\|\nabla u\|_{L^{2}}^{2}\|\nabla \theta\|_{L^{2}}\left\|\nabla^{2} \theta\right\|_{L^{2}} d s+C m_{0}^{\frac{1}{4}}+C m_{0}\left(t_{2}-t_{1}\right) \\
\leq & C\left(m_{0}^{\frac{1}{8}}+m_{0}^{\frac{1}{4}}\right)+C\left(1+\sup _{0 \leq t \leq T} \sigma\|\nabla u\|_{L^{2}}^{2}+m_{0}\right) \int_{t_{1}}^{t_{2}} \sigma\|\sqrt{\rho} \dot{u}\|_{L^{2}}^{2} d s \\
& +C\left(\sup _{0 \leq t \leq T} \sigma\|\nabla u\|_{L^{2}}^{2}+m_{0}\right) \int_{t_{1}}^{t_{2}} m_{0}^{\frac{1}{2}}\|\nabla \theta\|_{L^{2}}^{2} d s \\
& +C \sup _{0 \leq t \leq T} \sigma\|\nabla u\|_{L^{2}}^{2} K_{1} \int_{t_{1}}^{t_{2}} m_{0}^{\frac{1}{2}}\left\|\nabla^{2} \theta\right\|_{L^{2}}^{2} d s+C m_{0}\left(t_{2}-t_{1}\right) \\
\leq & C m_{0}^{\frac{1}{8}}+C\left(1+m_{0}^{\frac{1}{8}}+m_{0}\right) \int_{t_{1}}^{t_{2}} \sigma\|\sqrt{\rho} \dot{u}\|_{L^{2}}^{2} d s \\
& +C\left(m_{0}^{\frac{1}{8}}+m_{0}\right) m_{0}^{\frac{1}{2}}+C m_{0}^{\frac{5}{8}} K_{1}+C m_{0}\left(t_{2}-t_{1}\right) \\
\leq & C m_{0}^{\frac{1}{8}}+C\left(1+m_{0}^{\frac{1}{8}}+m_{0}\right) \int_{t_{1}}^{t_{2}} \sigma\|\sqrt{\rho} \dot{u}\|_{L^{2}}^{2} d s+C m_{0}\left(t_{2}-t_{1}\right),
\end{aligned}
$$

where we have used (3.1), (3.6), and (3.28).

In order to estimate the second term on the right-hand side of the above inequality, taking $\eta=\sigma$ in (3.23), integrating (3.23) over $\left(t_{1}, t_{2}\right) \in[0, T]$, we get

$$
\begin{aligned}
& \sigma \int \mu|\nabla u|^{2} d x+\int_{t_{1}}^{t_{2}} \int \sigma \rho|\dot{u}|^{2} d x d s \\
& \leq \mu \sigma\left\|\nabla u\left(t_{1}\right)\right\|_{L^{2}}^{2}+\sigma\|P\|_{L^{2}}\|\nabla u\|_{L^{2}}+C\left(m_{0}+m_{0}^{2}\right)\left(t_{2}-t_{1}\right)+C m_{0}^{\frac{1}{4}} \\
& \quad+C \int_{i-1}^{t} \sigma\|\nabla u\|_{L^{2}}^{4} d s+C \int_{i-1}^{t} m_{0}^{\frac{1}{2}}\|\nabla \theta\|_{L^{2}}^{2} d s
\end{aligned}
$$




$$
\begin{aligned}
\leq & \frac{1}{2} \sigma \mu\|\nabla u\|_{L^{2}}^{2}+C\left(m_{0}+m_{0}^{2}\right)\left(t_{2}-t_{1}\right) \\
& +C\left(m_{0}^{\frac{1}{4}}+m_{0}^{\frac{1}{8}}+m_{0}^{\frac{1}{2}}\right)+C \sup _{0 \leq t \leq T} \sigma\|\nabla u\|_{L^{2}}^{2} \int_{i-1}^{t}\|\nabla u\|_{L^{2}}^{2} d s \\
\leq & \frac{1}{2} \sigma \mu\|\nabla u\|_{L^{2}}^{2}+C\left(m_{0}+m_{0}^{2}\right)\left(t_{2}-t_{1}\right) \\
& +C\left(m_{0}^{\frac{1}{4}}+m_{0}^{\frac{1}{8}}+m_{0}^{\frac{1}{2}}\right)+C \sup _{0 \leq t \leq T} \sigma\|\nabla u\|_{L^{2}}^{2} m_{0}^{\frac{1}{4}} \\
\leq & \frac{1}{2} \sigma \mu\|\nabla u\|_{L^{2}}^{2}+C\left(m_{0}+m_{0}^{2}\right)\left(t_{2}-t_{1}\right)+C\left(m_{0}^{\frac{1}{4}}+m_{0}^{\frac{1}{8}}+m_{0}^{\frac{3}{8}}+m_{0}^{\frac{1}{2}}\right),
\end{aligned}
$$

which deduces

$$
\sigma \int \mu|\nabla u|^{2} d x+\int_{t_{1}}^{t_{2}} \int \sigma \rho|\dot{u}|^{2} d x d s \leq C m_{0}\left(t_{2}-t_{1}\right)+C m_{0}^{\frac{1}{8}}
$$

Then, inserting (3.39) into (3.37), (3.29) can be obtained. This completes the proof of Lemma 2.3.

Inspired by the methods in references [18, 42], in the following lemma, we use the Zlotnik inequality to prove the uniform upper bound of the density $\rho$.

Lemma 3.5 Under the condition of Proposition 3.1, it holds that

$$
\rho \leq \frac{3}{2} \bar{\rho}+1
$$

provided there exist constants $\varepsilon_{3}^{*}, \varepsilon_{4}^{*}$, and $\varepsilon_{5}^{*}$ such that $m_{0} \leq \min \left\{\varepsilon_{1}^{*}, \varepsilon_{2}^{*}, \varepsilon_{3}^{*}, \varepsilon_{4}^{*}, \varepsilon_{5}^{*}\right\}$.

Proof For any given $(x, t) \in \Omega \times[0, T]$. Denote $X(s ; x, t)$ is the solution to the initial value problem

$$
\left\{\begin{array}{l}
\frac{d}{d s} X(s ; x, t)=u(X(s ; x, t), s), \quad 0 \leq s<t, \\
X(t ; x, t)=x .
\end{array}\right.
$$

It is easy to verify that

$$
\frac{d}{d s} \rho(X(s ; x, t), s)+\rho(X(s ; x, t), s) \operatorname{div} u(X(s ; x, t), s)=0
$$

due to $(1.2)_{1}$. This gives

$$
Y^{\prime}(s)=g(s)+b^{\prime}(s)
$$

where

$$
\begin{aligned}
& Y(s)=\rho(X(s ; x, t), s), \quad g(s)=-\frac{A \rho^{\gamma+1}(X(s ; x, t), s)}{\mu}, \\
& b(s)=-\int_{0}^{s} \rho(X(s ; x, t), s)\left(\frac{C(t)}{\mu}+\operatorname{div} w(X(s ; x, t), s)\right) d s,
\end{aligned}
$$

and $C(t)=\mu \operatorname{div} v-P$. 
Next, we use Lemma 2.5 to prove the uniform upper bound of density

$$
b\left(t_{2}\right)-b\left(t_{1}\right)=\int_{t_{1}}^{t_{2}}\left\|\frac{\rho C(t)}{\mu}\right\|_{\infty} d s+\int_{t_{1}}^{t_{2}}\|\rho \operatorname{div} w\|_{\infty} d s:=K_{1}+K_{2} .
$$

In the following, we estimate the terms on the right-hand side of equation (3.42) one by one. In order to estimate $C(t)$, from equation (1.12), we have

$$
\nabla(\mu \operatorname{div} v-P)-\mu \nabla \times(\nabla \times v)=0 .
$$

Since $(\nabla \times(\nabla \times v))=\left(\partial_{2}\left(\partial_{1} v^{2}-\partial_{2} v^{1}\right),-\partial_{1}\left(\partial_{1} v^{2}-\partial_{2} v^{1}\right)\right)$ and the boundary condition (1.7) implies

$$
\begin{cases}v^{1}=\partial_{2} v^{2}=0, & x_{1}=0,1 \\ v^{2}=\partial_{1} v^{1}=0, & x_{2}=0,1 .\end{cases}
$$

Then we have $(\nabla \times(\nabla \times v)) \cdot n=0$ a.e. on $\partial \Omega$ and $\operatorname{div}(\nabla \times(\nabla \times v))=0$. Multiplying (3.43) by $\nabla(\mu \operatorname{div} v-P)$ and integrating the resulting equation over $\Omega$, we arrive at

$$
\|\nabla(\mu \operatorname{div} v-P)\|_{L^{2}}=0
$$

which implies that there exists $C(t)$ such that

$$
C(t)=\mu \operatorname{div} v-P .
$$

Using (1.12), we have $\|\nabla v\|_{L^{2}} \leq C\|P\|_{L^{2}}$. Integrating (3.45) over the square domain, we get

$$
C(t) \leq C\left(\|\nabla v\|_{L^{2}}+\|P\|_{L^{2}}\right) \leq C\|P\|_{L^{2}} \leq C \bar{\rho}^{\frac{2 \gamma-1}{2}} m_{0}^{\frac{1}{2}} .
$$

Then we have

$$
K_{1}=\int_{t_{1}}^{t_{2}}\left\|\frac{\rho C(t)}{\mu}\right\|_{\infty} d s \leq \frac{C \bar{\rho}^{\frac{2 \gamma+1}{2}} m_{0}^{\frac{1}{2}}}{\mu}\left(t_{2}-t_{1}\right) \leq \frac{1}{4 \mu}\left(t_{2}-t_{1}\right),
$$

provided there exists a constant $\varepsilon_{3}^{*}, m_{0} \leq \varepsilon_{3}^{*}$, such that

$$
C \bar{\rho}^{\frac{2 \gamma+1}{2}} m_{0}^{\frac{1}{2}} \leq \frac{1}{4}
$$

In order to estimate $K_{2}$, we consider the following three cases:

(1) $0 \leq t_{1} \leq t_{2} \leq \sigma(T)$.

$$
\begin{aligned}
K_{2} & =\int_{t_{1}}^{t_{2}}\|\rho \operatorname{div} w\|_{L^{\infty}} d s \leq C \int_{0}^{\sigma(T)}\|\nabla w\|_{L^{2}}^{\frac{1}{3}}\|\nabla w\|_{W^{1,4}}^{\frac{2}{3}} d s \\
& \leq C \int_{0}^{\sigma(T)}\left(\|\nabla u\|_{L^{2}}+\|P\|_{L^{2}}\right)^{\frac{1}{3}}\left(\|\nabla \dot{u}\|_{L^{2}}+\|\nabla \theta\|_{L^{2}}\right)^{\frac{2}{3}} d s \\
& \leq C \int_{0}^{\sigma(T)} \sigma^{-\frac{1}{2}}\left(\sigma^{\frac{1}{2}}\|\nabla u\|_{L^{2}}+\sigma^{\frac{1}{2}}\|P\|_{L^{2}}\right)^{\frac{1}{3}}\left(\sigma\|\nabla \dot{u}\|_{L^{2}}^{2}+\sigma\|\nabla \theta\|_{L^{2}}^{2}\right)^{\frac{1}{3}} d s
\end{aligned}
$$




$$
\begin{aligned}
\leq & C \int_{0}^{\sigma(T)} \sigma^{-\frac{1}{2}}\left(m_{0}^{\frac{1}{16}}+\sigma^{\frac{1}{2}} \bar{\rho}^{\frac{2 \gamma-1}{2}} m_{0}^{\frac{1}{2}}\right)^{\frac{1}{3}}\left(\sigma\|\nabla \dot{u}\|_{L^{2}}^{2}+\sigma\|\nabla \theta\|_{L^{2}}^{2}\right)^{\frac{1}{3}} d s \\
\leq & C\left(m_{0}^{\frac{1}{16}}+\bar{\rho}^{\frac{2 \gamma-1}{2}} m_{0}^{\frac{1}{2}}\right)^{\frac{1}{3}}\left(\int_{0}^{\sigma(T)} \sigma^{-\frac{3}{4}} d s\right)^{\frac{2}{3}} \\
& \times\left(\int_{0}^{\sigma(T)}\left(\sigma\|\nabla \dot{u}\|_{L^{2}}^{2}+\sigma\|\nabla \theta\|_{L^{2}}^{2}\right) d s\right)^{\frac{1}{3}} \\
\leq & C\left(m_{0}^{\frac{1}{16}}+\bar{\rho}^{\frac{2 \gamma-1}{2}} m_{0}^{\frac{1}{2}}\right)^{\frac{1}{3}}\left(\int_{0}^{\sigma(T)} \sigma\|\nabla \dot{u}\|_{L^{2}}^{2} d s+1\right)^{\frac{1}{3}},
\end{aligned}
$$

where we have used Lemma 3.3. It remains to estimate the term on the right-hand side of inequality (3.48). To do this, we take $\eta=\sigma$ in (3.35) and integrate over $(0, \sigma(T))$, then we have

$$
\begin{aligned}
& \int \sigma \rho|\dot{u}|^{2} d x+\int_{0}^{\sigma(T)} \mu \sigma\|\nabla \dot{u}\|_{L^{2}}^{2} d s \\
& \leq C+\int_{0}^{\sigma(T)}\left|\sigma^{\prime}\right| \int \rho|\dot{u}|^{2} d x d s+C \int_{0}^{\sigma(T)} \sigma\|\nabla u\|_{L^{2}}^{2} d s \\
& \quad+C \int_{0}^{\sigma(T)} \sigma\left(\|\nabla u\|_{L^{2}}^{2}+m_{0}\right)\left(\|\sqrt{\rho} \dot{u}\|_{L^{2}}^{2}+m_{0}^{\frac{1}{2}}\|\nabla \theta\|_{L^{2}}^{2}\right) d s \\
& \quad+C \int_{0}^{\sigma(T)} \sigma m_{0}^{\frac{1}{2}}\|\nabla u\|_{L^{2}}^{2}\|\nabla \theta\|_{L^{2}}\left\|\nabla^{2} \theta\right\|_{L^{2}} d s+C \sigma m_{0} \\
& \leq C\left(1+K+m_{0}^{\frac{1}{4}}\right)+C\left(m_{0}^{\frac{1}{8}}+m_{0}\right)(K+1)+C\left(m_{0}^{\frac{5}{8}}+m_{0}\right) \\
& \leq C .
\end{aligned}
$$

From (3.48) and (3.49), we can see that

$$
K_{2} \leq C\left(m_{0}^{\frac{1}{16}}+\bar{\rho}^{\frac{2 \gamma-1}{2}} m_{0}^{\frac{1}{2}}\right)^{\frac{1}{3}} \leq \frac{1}{8} \bar{\rho}
$$

provided there exists a constant $\varepsilon_{4}^{*}$ such that $m_{0} \leq \varepsilon_{4}^{*}$.

(2) $\sigma(T) \leq t_{1} \leq t_{2} \leq T$.

$$
\begin{aligned}
K_{2} \leq & \frac{1}{4 \mu}\left(t_{2}-t_{1}\right)+4 \mu \int_{t_{1}}^{t_{2}}\|\rho \operatorname{div} w\|_{L^{\infty}}^{2} d s \\
\leq & \frac{1}{4 \mu}\left(t_{2}-t_{1}\right)+4 \bar{\rho}^{2} \mu \int_{t_{1}}^{t_{2}}\|\operatorname{div} w\|_{L^{\infty}}^{2} d s \\
\leq & \frac{1}{4 \mu}\left(t_{2}-t_{1}\right)+C \bar{\rho}^{2} \int_{t_{1}}^{t_{2}}\|\nabla w\|_{L^{2}}^{\frac{2}{3}}\|\nabla w\|_{W^{1,4}}^{\frac{4}{3}} d s \\
\leq & \frac{1}{4 \mu}\left(t_{2}-t_{1}\right)+C \bar{\rho}^{2} \int_{t_{1}}^{t_{2}}\left(\|\nabla u\|_{L^{2}}+\|P\|_{L^{2}}\right)^{\frac{2}{3}}\left(\|\nabla \dot{u}\|_{L^{2}}+\|\nabla \theta\|_{L^{2}}\right)^{\frac{4}{3}} d s \\
\leq & \frac{1}{4 \mu}\left(t_{2}-t_{1}\right)+C \bar{\rho}^{2}\left(\int_{t_{1}}^{t_{2}}\left(\|\nabla u\|_{L^{2}}^{2}+\|P\|_{L^{2}}^{2}\right) d s\right)^{\frac{1}{3}} \\
& \times\left(\int_{t_{1}}^{t_{2}}\left(\|\nabla \dot{u}\|_{L^{2}}^{2}+\|\nabla \theta\|_{L^{2}}^{2}\right) d s\right)^{\frac{2}{3}}
\end{aligned}
$$




$$
\begin{aligned}
\leq & \frac{1}{4 \mu}\left(t_{2}-t_{1}\right)+C \bar{\rho}^{2}\left(m_{0}^{\frac{1}{4}}+\bar{\rho}^{2 \gamma-1} m_{0}\left(t_{2}-t_{1}\right)\right)^{\frac{1}{3}} \\
& \times\left(\int_{t_{1}}^{t_{2}}\left(\|\nabla \dot{u}\|_{L^{2}}^{2}+\|\nabla \theta\|_{L^{2}}^{2}\right) d s\right)^{\frac{2}{3}} \\
\leq & \frac{1}{4 \mu}\left(t_{2}-t_{1}\right)+C m_{0}^{\frac{1}{12}}\left(\int_{t_{1}}^{t_{2}}\|\nabla \dot{u}\|_{L^{2}}^{2} d s+1\right)^{\frac{2}{3}} \\
& +C m_{0}^{\frac{1}{3}}\left(t_{2}-t_{1}\right)^{\frac{1}{3}}\left(\int_{t_{1}}^{t_{2}}\|\nabla \dot{u}\|_{L^{2}}^{2} d s+1\right)^{\frac{2}{3}} \\
\leq & \frac{1}{4 \mu}\left(t_{2}-t_{1}\right)+C \int_{t_{1}}^{t_{2}}\|\nabla \dot{u}\|_{L^{2}}^{2} d s+C\left(m_{0}^{\frac{1}{4}}+m_{0}^{\frac{1}{12}}\right) \\
& +C m_{0}\left(t_{2}-t_{1}\right)+C m_{0}^{\frac{1}{3}}\left(t_{2}-t_{1}\right)^{\frac{1}{3}} \\
\leq & \frac{1}{4 \mu}\left(t_{2}-t_{1}\right)+C\left(m_{0}+m_{0}^{\frac{1}{4}}\right)\left(t_{2}-t_{1}\right)+C\left(m_{0}^{\frac{1}{4}}+m_{0}^{\frac{1}{8}}+m_{0}^{\frac{3}{8}}+m_{0}^{\frac{1}{12}}\right)
\end{aligned}
$$

where in the last inequality we have used (3.29). Then we get

$$
K_{2} \leq \frac{1}{2 \mu}\left(t_{2}-t_{1}\right)+\frac{1}{8} \bar{\rho}
$$

provided there exists a constant $\varepsilon_{5}^{*}, m_{0} \leq \varepsilon_{5}^{*}$, such that

$$
C\left(m_{0}+m_{0}^{\frac{1}{4}}\right) \leq \frac{1}{4 \mu}, \quad C\left(m_{0}^{\frac{1}{4}}+m_{0}^{\frac{1}{8}}+m_{0}^{\frac{3}{8}}+m_{0}^{\frac{1}{12}}\right) \leq \frac{1}{8} \bar{\rho} .
$$

(3) $0 \leq t_{1} \leq \sigma(T) \leq t_{2} \leq T$.

Combining case (1) and case (2), we can easily obtain

$$
K_{2}=\int_{t_{1}}^{\sigma(T)}\|\rho \operatorname{div} w\|_{L^{\infty}} d s+\int_{\sigma(T)}^{t_{2}}\|\rho \operatorname{div} w\|_{L^{\infty}} d s \leq \frac{1}{2 \mu}\left(t_{2}-t_{1}\right)+\frac{1}{4} \bar{\rho} .
$$

Taking (3.42), (3.47), (3.50), (3.52), and (3.53) into consideration, we have

$$
\left|b\left(t_{2}\right)-b\left(t_{1}\right)\right| \leq \frac{1}{\mu}\left(t_{2}-t_{1}\right)+\frac{1}{2} \bar{\rho},
$$

provided there exist constants $\varepsilon_{3}^{*}, \varepsilon_{4}^{*}$, and $\varepsilon_{5}^{*}$ as mentioned above such that $m_{0} \leq$ $\min \left\{\varepsilon_{1}, \varepsilon_{1}^{*}, \varepsilon_{2}^{*}, \varepsilon_{3}^{*}, \varepsilon_{4}^{*}, \varepsilon_{5}^{*}\right\}$.

Then we can choose $N_{0}, N_{1}$ as follows:

$$
N_{0}=\frac{1}{2} \bar{\rho}, \quad N_{1}=\frac{1}{\mu} .
$$

Choosing $\xi^{*}=\bar{\rho}+1$, we can see that

$$
g(\xi)=-\frac{\xi^{\gamma+1}}{\mu} \leq-\frac{1}{\mu}=-N_{1} \quad \text { for } \xi \geq \xi^{*}
$$


Using Lemma 2.5, we obtain

$$
\sup _{t \in[0, T]}\|\rho\|_{L^{\infty}} \leq \max \left\{\rho_{0}, \xi^{*}\right\}+N_{0} \leq \max \{\bar{\rho}, \bar{\rho}+1\}+N_{0} \leq \bar{\rho}+1+\frac{1}{2} \bar{\rho}=\frac{3}{2} \bar{\rho}+1 .
$$

This completes the proof of Lemma 3.5.

Combining Lemmas 3.2-3.5, if we take $m_{0} \leq \min \left\{\varepsilon_{1}, \varepsilon_{1}^{*}, \varepsilon_{2}^{*}, \varepsilon_{3}^{*}, \varepsilon_{4}^{*}, \varepsilon_{5}^{*}\right\}$, then Proposition 3.1 is proved.

\section{Time-dependent higher-order estimates}

For completeness of our proof, we give the time-dependent higher-order estimates of the solution $(\rho, u, \theta)$ in what follows. First, we prove the second-order a priori estimates in Lemma 4.1 for $u$ and Lemma 4.2 for $(\rho, \theta)$, respectively, which can be derived in a similar manner as those obtained in $[18,22]$ after some modifications. In the rest of this paper, the constant $C$ may depend on time $T$.

Lemma 4.1 Let $(\rho, u, \theta)$ be a strong solution of (1.2), (1.6)-(1.7) on $\Omega \times(0, T]$, under the condition of Theorem 1.1, it holds that

$$
\begin{aligned}
& \sup _{0 \leq t \leq T}\|\sqrt{\rho} \dot{u}\|_{L^{2}}^{2}+\int_{0}^{T}\|\nabla \dot{u}\|_{L^{2}}^{2} d s \leq C(T), \\
& \sup _{0 \leq t \leq T}\left(\|\nabla \rho\|_{L^{p}}+\|u\|_{H^{2}}\right) \leq C(T) .
\end{aligned}
$$

Proof Taking $\eta=1$ in (3.35), integrating (3.35) over $(0, \sigma(T)]$, we get

$$
\begin{aligned}
& \sup _{0 \leq t \leq \sigma(T)}\|\sqrt{\rho} \dot{u}\|_{L^{2}}^{2}+\int_{0}^{\sigma(T)} \mu\|\nabla \dot{u}\|_{L^{2}}^{2} d s \\
& \leq C\left(1+m_{0}^{\frac{1}{4}}+m_{0}\right)+C \int_{0}^{\sigma(T)}\left(\|\nabla u\|_{L^{2}}^{2}+m_{0}\right)\left(\|\sqrt{\rho} \dot{u}\|_{L^{2}}^{2}+m_{0}^{\frac{1}{2}}\|\nabla \theta\|_{L^{2}}^{2}\right) d s \\
& \quad+C \int_{0}^{\sigma(T)} m_{0}^{\frac{1}{2}}\|\nabla u\|_{L^{2}}^{2}\|\nabla \theta\|_{L^{2}}\left\|\nabla^{2} \theta\right\|_{L^{2}} d s \\
& \leq C,
\end{aligned}
$$

from which, combining (3.28) and (3.29), we obtain

$$
\sup _{0 \leq t \leq T}\|\sqrt{\rho} \dot{u}\|_{L^{2}}^{2}+\int_{0}^{T}\|\nabla \dot{u}\|_{L^{2}}^{2} d t \leq C(T) .
$$

Next, applying the operator $\nabla$ to $(1.1)_{1}$ and multiplying the resulting equation by $p|\nabla \rho|^{p-2} \nabla \rho, p>2$, we obtain

$$
\begin{aligned}
\frac{d}{d t} \int|\nabla \rho|^{p} d x= & (1-p) \int|\nabla \rho|^{p} \operatorname{div} u d x-p \int|\nabla \rho|^{p-2} \nabla \rho(\nabla u \cdot \nabla \rho) d x \\
& -p \int \rho|\nabla \rho|^{p-2} \nabla \rho \cdot \nabla(\operatorname{div} u) d x
\end{aligned}
$$




$$
\begin{aligned}
& \leq C \int|\nabla \rho|^{p}|\nabla u| d x+C \int|\nabla \rho|^{p-1}\left|\nabla^{2} u\right| d x \\
& \leq C\|\nabla u\|_{L^{\infty}}\|\nabla \rho\|_{L^{p}}^{p}+C\|\nabla \rho\|_{L^{p}}^{p-1}\left\|\nabla^{2} u\right\|_{L^{p}} .
\end{aligned}
$$

Then we have

$$
\frac{d}{d t}\|\nabla \rho\|_{p} \leq C\left(\|\nabla u\|_{L^{\infty}}\|\nabla \rho\|_{L^{p}}+\left\|\nabla^{2} u\right\|_{L^{p}}\right)
$$

where the terms on the right-hand side can be estimated as follows:

$$
\begin{aligned}
\|\nabla u\|_{L^{\infty}} & \leq C\left(\|\nabla w\|_{L^{\infty}}+\|\nabla v\|_{L^{\infty}}\right) \\
& \leq C\left(\|\nabla w\|_{L^{p}}+\left\|\nabla^{2} w\right\|_{L^{p}}+\|\nabla v\|_{L^{\infty}}\right) \\
& \leq C\left(\|\nabla \dot{u}\|_{L^{2}}+\|\nabla \theta\|_{L^{2}}+\|\nabla v\|_{\mathrm{BMO}} \ln \left(e+\left\|\nabla^{2} v\right\|_{L^{p}}\right)+1\right) \\
& \leq C\left(\|\nabla \dot{u}\|_{L^{2}}+\left(\|P\|_{L^{\infty}}+\|P\|_{L^{2}}\right) \ln \left(e+\left\|\nabla^{2} v\right\|_{L^{p}}\right)+1\right) \\
& \leq C\left(\|\nabla \dot{u}\|_{L^{2}}+\ln \left(e+\|\nabla \rho\|_{L^{p}}\right)+1\right)
\end{aligned}
$$

and

$$
\begin{aligned}
\left\|\nabla^{2} u\right\|_{L^{p}} & \leq C\left(\left\|\nabla^{2} v\right\|_{L^{p}}+\left\|\nabla^{2} w\right\|_{L^{p}}\right) \leq C\left(\|\nabla P\|_{L^{p}}+\|\rho \dot{u}\|_{L^{p}}+\|\theta\|_{L^{p}}\right) \\
& \leq C\left(\|\nabla \rho\|_{L^{p}}+\|\nabla \dot{u}\|_{L^{2}}\right) .
\end{aligned}
$$

Inserting (4.7) and (4.8) into (4.6), we have

$$
\begin{aligned}
\frac{d}{d t}\left(\|\nabla \rho\|_{L^{p}}+e\right) \leq & C\left(\|\nabla \dot{u}\|_{L^{2}}+e\right) \ln \left(e+\|\nabla \rho\|_{L^{p}}\right)\|\nabla \rho\|_{L^{p}} \\
& +C\left(\|\nabla \dot{u}\|_{L^{2}}+e\right)\left(\|\nabla \rho\|_{L^{p}}+e\right) .
\end{aligned}
$$

Both sides of (4.9) divided by $\|\nabla \rho\|_{p}+e$ lead to

$$
\frac{d}{d t} \ln \left(\|\nabla \rho\|_{L^{p}}+e\right) \leq C\left(\|\nabla \dot{u}\|_{L^{2}}+e\right) \ln \left(e+\|\nabla \rho\|_{L^{p}}\right)+C\left(\|\nabla \dot{u}\|_{L^{2}}+e\right) .
$$

Then, by using Gronwall's inequality and (4.4), it holds that

$$
\sup _{0 \leq t \leq T}\|\nabla \rho\|_{L^{p}} \leq C(T) .
$$

Moreover, from (4.4), we have

$$
\|u\|_{H^{2}} \leq C\left(\|\rho \dot{u}\|_{L^{2}}+\|\nabla P\|_{L^{2}}+\|\theta\|_{L^{2}}\right) \leq C\left(\|\rho \dot{u}\|_{L^{2}}+\|\nabla \rho\|_{L^{p}}+1\right) \leq C(T) .
$$

This completes the proof of Lemma 4.1.

Lemma 4.2 Let $(\rho, u, \theta)$ be a classical solution of $(1.2),(1.6)-(1.7)$ on $\Omega \times(0, T]$, under the condition of Theorem 1.1, the following estimates hold:

$$
\sup _{0 \leq t \leq T}\left\|\sqrt{\rho} u_{t}\right\|_{L^{2}}^{2}+\int_{0}^{T}\left\|\nabla u_{t}\right\|_{L^{2}}^{2} d s \leq C(T)
$$




$$
\begin{aligned}
& \sup _{0 \leq t \leq T}\left(\|\rho\|_{H^{2}}^{2}+\|P(\rho)\|_{H^{2}}^{2}\right) \leq C(T), \\
& \sup _{0 \leq t \leq T}\left(\left\|\nabla^{2} \theta\right\|_{L^{2}}^{2}+\left\|\theta_{t}\right\|_{L^{2}}^{2}\right)+\int_{0}^{T}\left\|\nabla^{3} \theta\right\|_{L^{2}}^{2} d s \leq C(T) .
\end{aligned}
$$

Proof Inequality (4.13) follows directly from the following simple fact:

$$
\begin{aligned}
\int \rho\left|u_{t}\right|^{2} d x & \leq \int \rho|\dot{u}|^{2} d x+\int \rho|u \cdot \nabla u|^{2} d x \\
& \leq C+\|\sqrt{\rho} u\|_{L^{2}}\|u\|_{L^{6}}\|\nabla u\|_{L^{6}}^{2} \leq C,
\end{aligned}
$$

and

$$
\begin{aligned}
\int_{0}^{T}\left\|\nabla u_{t}\right\|_{L^{2}}^{2} d s & \leq \int_{0}^{T}\|\nabla \dot{u}\|_{L^{2}}^{2} d s+\int_{0}^{T}\|\nabla(u \cdot \nabla u)\|_{L^{2}}^{2} d s \\
& \leq C+\int_{0}^{T}\|u\|_{L^{\infty}}^{2}\left\|\nabla^{2} u\right\|_{L^{2}}^{2} d s+\int_{0}^{T}\|\nabla u\|_{L^{4}}^{4} d s \\
& \leq C
\end{aligned}
$$

where in the last inequality we have used Sobolev embedding inequalities and Lemma 4.1. Next, we prove (4.14). Since $P$ satisfies

$$
P_{t}+u \cdot \nabla P+\gamma P \operatorname{div} u=0
$$

which together with (1.2) 1 yields that

$$
\begin{aligned}
& \frac{d}{d t}\left(\left\|\nabla^{2} \rho\right\|_{L^{2}}^{2}+\|\nabla P\|_{L^{2}}^{2}\right) \\
& \leq C\left(\left\|\nabla^{2} u\right\|_{L^{4}}\|\nabla \rho\|_{L^{4}}\left\|\nabla^{2} \rho\right\|_{L^{2}}+\|\nabla u\|_{L^{\infty}}\left\|\nabla^{2} \rho\right\|_{L^{2}}^{2}+\left\|\nabla^{2} u\right\|_{L^{4}}\|\nabla P\|_{L^{4}}\left\|\nabla^{2} P\right\|_{L^{2}}\right. \\
& \left.+\|\nabla u\|_{L^{\infty}}\left\|\nabla^{2} P\right\|_{L^{2}}^{2}+\left\|\nabla^{3} u\right\|_{L^{2}}\left\|\nabla^{2} P\right\|_{L^{2}}\right) \\
& \leq C\left(\left\|\nabla^{2} u\right\|_{L^{2}}^{\frac{1}{2}}\left\|\nabla^{3} u\right\|_{L^{2}}^{\frac{1}{2}}\|\nabla \rho\|_{L^{2}}^{\frac{1}{2}}\left\|\nabla^{2} \rho\right\|_{L^{2}}^{\frac{3}{2}}+\|\nabla u\|_{L^{\infty}}\left\|\nabla^{2} \rho\right\|_{L^{2}}^{2}\right. \\
& \left.+\left\|\nabla^{2} u\right\|_{L^{2}}^{\frac{1}{2}}\left\|\nabla^{3} u\right\|_{L^{2}}^{\frac{1}{2}}\|\nabla P\|_{L^{2}}^{\frac{1}{2}}\left\|\nabla^{2} P\right\|_{L^{2}}^{\frac{3}{2}}+\|\nabla u\|_{L^{\infty}}\left\|\nabla^{2} P\right\|_{L^{2}}^{2}+\left\|\nabla^{3} u\right\|_{L^{2}}\left\|\nabla^{2} P\right\|_{L^{2}}\right) \\
& \leq C\left(\left\|\nabla^{3} u\right\|_{L^{2}}^{2}+\left\|\nabla^{2} \rho\right\|_{L^{2}}^{2}+\left\|\nabla^{2} P\right\|_{L^{2}}^{2}+\|\nabla u\|_{L^{\infty}}\left\|\nabla^{2} \rho\right\|_{L^{2}}^{2}+\|\nabla u\|_{L^{\infty}}\left\|\nabla^{2} P\right\|_{L^{2}}^{2}\right) \\
& \leq C\left\|\nabla^{3} u\right\|_{L^{2}}^{2}+C\left(\|\nabla u\|_{L^{\infty}}+1\right)\left(\left\|\nabla^{2} \rho\right\|_{L^{2}}^{2}+\left\|\nabla^{2} P\right\|_{L^{2}}^{2}\right) \\
& \leq C\left(\|\nabla \dot{u}\|_{L^{2}}+1\right)+C\left(\|\nabla \dot{u}\|_{L^{2}}+\ln \left(e+\|\nabla \rho\|_{L^{p}}\right)+1\right)\left(\left\|\nabla^{2} \rho\right\|_{L^{2}}^{2}+\left\|\nabla^{2} P\right\|_{L^{2}}^{2}\right),
\end{aligned}
$$

where in the last inequality we have used (4.7). From the following standard $L^{2}$-estimate, for elliptic system (1.12) and (1.13), we obtain

$$
\begin{aligned}
\left\|\nabla^{3} u\right\|_{L^{2}} & \leq C\left(\left\|\nabla^{3} v\right\|_{L^{2}}+\left\|\nabla^{3} w\right\|_{L^{2}}\right) \leq C\left(\left\|\nabla^{2} P\right\|_{L^{2}}+\|\nabla(\rho \dot{u})\|_{L^{2}}+\|\nabla \theta\|_{L^{2}}\right) \\
& \leq C\left(\left\|\nabla^{2} \rho\right\|_{L^{2}}+\|\nabla \dot{u}\|_{L^{2}}+1\right) .
\end{aligned}
$$

Then, combining (4.19), Lemma 4.1, and Gronwall's inequality, we have (4.14). 
In order to estimate the second-order derivative of temperature, we apply the operator $\nabla^{2}$ on both sides of equation $(1.2)_{3}$, then we have

$$
\begin{aligned}
& \frac{1}{2} \frac{d}{d t}\left\|\nabla^{2} \theta\right\|_{L^{2}}^{2}+\kappa\left\|\nabla^{3} \theta\right\|_{L^{2}}^{2} \\
& \quad=-\int \nabla^{2}(u \cdot \nabla) \theta \nabla^{2} \theta d x \\
& \quad=-\int\left(\nabla^{2} u \cdot \nabla\right) \theta \nabla^{2} \theta d x-\int\left(u \cdot \nabla^{3}\right) \theta \nabla^{2} \theta d x-2 \int\left(\nabla u \cdot \nabla^{2}\right) \theta \nabla^{2} \theta d x \\
& \quad \leq\left\|\nabla^{2} u\right\|_{L^{2}}\|\nabla \theta\|_{L^{4}}\left\|\nabla^{2} \theta\right\|_{L^{4}}+\|u\|_{L^{\infty}}\left\|\nabla^{3} \theta\right\|_{L^{2}}\left\|\nabla^{2} \theta\right\|_{L^{2}}+\|\nabla u\|_{L^{4}}\left\|\nabla^{2} \theta\right\|_{L^{2}}\left\|\nabla^{2} \theta\right\|_{L^{4}} \\
& \quad \leq C\left(\left\|\nabla^{2} \theta\right\|_{L^{2}}\left\|\nabla^{3} \theta\right\|_{L^{2}}^{\frac{1}{2}}+\left\|\nabla^{3} \theta\right\|_{L^{2}}\left\|\nabla^{2} \theta\right\|_{L^{2}}+\left\|\nabla^{3} \theta\right\|_{L^{2}}^{\frac{3}{2}}\left\|\nabla^{2} \theta\right\|_{L^{2}}^{\frac{1}{2}}\right) \\
& \quad \leq \frac{\kappa}{2}\left\|\nabla^{3} \theta\right\|_{L^{2}}^{2}+C\left(1+\left\|\nabla^{2} \theta\right\|_{L^{2}}^{2}\right),
\end{aligned}
$$

from which, after integration over time interval $(0, t)$ and together with $(1.2)_{3},(4.15)$ is proved.

Lemma 4.3 Let $(\rho, u, \theta)$ be a classical solution of $(1.2),(1.6)-(1.7)$ on $\Omega \times(0, T]$, under the condition of Theorem 1.1, the following estimates hold:

$$
\begin{aligned}
& \sup _{0 \leq t \leq T}\left(\left\|\rho_{t}\right\|_{H^{1}}+\left\|P_{t}\right\|_{H^{1}}\right)+\int_{0}^{T}\left(\left\|\rho_{t t}\right\|_{L^{2}}^{2}+\left\|P_{t t}\right\|_{L^{2}}^{2}\right) d s \leq C(T), \\
& \sup _{0 \leq t \leq T}\left\|\nabla u_{t}\right\|_{L^{2}}^{2}+\int_{0}^{T}\left\|\sqrt{\rho} u_{t t}\right\|_{L^{2}}^{2} d s \leq C(T) .
\end{aligned}
$$

Proof First, from (4.18) and Lemma 4.1, we obtain

$$
\left\|P_{t}\right\|_{L^{2}} \leq C\left(\|u\|_{L^{\infty}}\|\nabla P\|_{L^{2}}+\|\nabla u\|_{L^{2}}\right) \leq C
$$

Furthermore, differentiating (4.18) yields

$$
\nabla P_{t}+u \cdot \nabla \nabla P+\nabla u \cdot \nabla P+\gamma \nabla P \operatorname{div} u+\gamma P \nabla \operatorname{div} u=0,
$$

from which, together with Lemma 4.1 and Lemma 4.2, one gets

$$
\left\|\nabla P_{t}\right\|_{L^{2}} \leq C\left(\|u\|_{L^{\infty}}\left\|\nabla^{2} P\right\|_{L^{2}}+\|\nabla u\|_{L^{4}}\|\nabla P\|_{L^{4}}+\left\|\nabla^{2} u\right\|_{L^{2}}\right) \leq C .
$$

The combination of (4.23) and (4.25) implies

$$
\sup _{0 \leq t \leq T}\left\|P_{t}\right\|_{H^{1}} \leq C
$$

Note that $P_{t t}$ satisfies

$$
P_{t t}+u_{t} \cdot \nabla P+u \cdot \nabla P_{t}+\gamma P \operatorname{div} u_{t}+\gamma P_{t} \operatorname{div} u=0,
$$


from which, together with (4.26) and Lemma 4.2, we have

$$
\int_{0}^{T}\left\|P_{t t}\right\|_{L^{2}}^{2} d s \leq \int_{0}^{T} C\left(\left\|u_{t}\right\|_{L^{4}}\|\nabla P\|_{L^{4}}+\|\nabla u\|_{L^{4}}\left\|P_{t}\right\|_{L^{4}}+\left\|\nabla u_{t}\right\|_{L^{2}}\right)^{2} d s \leq C .
$$

Next, we differentiate $(1.2)_{2}$ with respect to $t$, then multiplying the resulting equation by $u_{t t}$, one gets after integration by parts that

$$
\begin{aligned}
\frac{1}{2} \frac{d}{d t} \int\left(\mu\left|\nabla u_{t}\right|^{2}\right) d x+\int \rho u_{t t}^{2} d x \\
=\frac{d}{d t}\left(-\frac{1}{2} \int \rho_{t}\left|u_{t}\right|^{2} d x-\int \rho_{t} u \cdot \nabla u \cdot u_{t} d x+\int P_{t} \operatorname{div} u_{t} d x+\int \rho_{t} \theta u_{t} d x\right) \\
\quad+\frac{1}{2} \int \rho_{t t}\left|u_{t}\right|^{2} d x+\int\left(\rho_{t} u \cdot \nabla u\right)_{t} \cdot u_{t} d x-\int \rho u_{t} \cdot \nabla u \cdot u_{t t} d x \\
\quad-\int \rho u \cdot \nabla u_{t} \cdot u_{t t} d x-\int P_{t t} \operatorname{div} u_{t} d x-\int \rho_{t t} \theta e_{2} \cdot u_{t} d x \\
\quad-\int \rho_{t} \theta_{t} e_{2} \cdot u_{t} d x+\int \rho \theta_{t} e_{2} \cdot u_{t t} d x \\
:=\frac{d}{d t} L_{0}+\sum_{i=1}^{8} L_{i} .
\end{aligned}
$$

The terms on the right-hand side of equation (4.29) can be estimated as follows:

$$
\begin{aligned}
L_{0}= & -\frac{1}{2} \int \rho_{t}\left|u_{t}\right|^{2} d x-\int \rho_{t} u \cdot \nabla u \cdot u_{t} d x+\int P_{t} \operatorname{div} u_{t} d x+\int \rho_{t} \theta u_{t} d x \\
\leq & C\left(\|u\|_{L^{\infty}}\left\|\sqrt{\rho} u_{t}\right\|_{L^{2}}\left\|\nabla u_{t}\right\|_{L^{2}}+\left\|\rho_{t}\right\|_{L^{4}}\|u\|_{L^{\infty}}\|\nabla u\|_{L^{4}}\left\|u_{t}\right\|_{L^{2}}+\left\|P_{t}\right\|_{L^{2}}\left\|\nabla u_{t}\right\|_{L^{2}}\right. \\
& \left.+\|\nabla \rho\|_{L^{2}}\|u\|_{L^{\infty}}\|\theta\|_{L^{\infty}}\left\|u_{t}\right\|_{L^{2}}+\|\nabla u\|_{L^{2}}\|\rho\|_{L^{\infty}}\|\theta\|_{L^{\infty}}\left\|u_{t}\right\|_{L^{2}}\right) \\
\leq & C\left(\|u\|_{H^{2}}\left\|\sqrt{\rho} u_{t}\right\|_{L^{2}}\left\|\nabla u_{t}\right\|_{L^{2}}+\left\|\rho_{t}\right\|_{H^{1}}\|u\|_{H^{2}}^{2}\left\|\nabla u_{t}\right\|_{L^{2}}+\left\|P_{t}\right\|_{L^{2}}\left\|\nabla u_{t}\right\|_{L^{2}}\right. \\
& \left.+\|\nabla \rho\|_{L^{2}}\left\|u_{t}\right\|_{L^{2}}+\|\nabla u\|_{L^{2}}\left\|u_{t}\right\|_{L^{2}}\right) \\
\leq & \delta\left\|\nabla u_{t}\right\|_{L^{2}}^{2}+C\left(\|u\|_{H^{2}}^{2}\left\|\sqrt{\rho} u_{t}\right\|_{L^{2}}^{2}+\left\|\rho_{t}\right\|_{H^{1}}^{2}\|u\|_{H^{2}}^{4}+\left\|P_{t}\right\|_{L^{2}}^{2}+\|\nabla \rho\|_{L^{2}}^{2}+\|\nabla u\|_{L^{2}}^{2}\right) \\
\leq & \delta\left\|\nabla u_{t}\right\|_{L^{2}}^{2}+C,
\end{aligned}
$$

where we have used Lemma 4.1, Lemma 4.2, (4.21), and the Poincaré inequality.

$$
\begin{aligned}
L_{1}= & \frac{1}{2} \int \rho_{t t}\left|u_{t}\right|^{2} d x=-\frac{1}{2} \int\left(\rho_{t} u+\rho u_{t}\right) \cdot \nabla\left|u_{t}\right|^{2} d x \\
\leq & C\left(\left\|\rho_{t}\right\|_{L^{4}}\|u\|_{L^{\infty}}\left\|u_{t}\right\|_{L^{4}}\left\|\nabla u_{t}\right\|_{L^{2}}+\left\|u_{t}\right\|_{L^{4}}^{2}\left\|\nabla u_{t}\right\|_{L^{2}}\right) \\
\leq & C\left(1+\left\|\nabla u_{t}\right\|_{L^{2}}^{2}\right)\left\|\nabla u_{t}\right\|_{L^{2}}^{2}, \\
L_{2}= & \int\left(\rho_{t} u \cdot \nabla u\right)_{t} \cdot u_{t} d x=\int\left(\rho_{t t} u \cdot \nabla u+\rho_{t} u_{t} \cdot \nabla u+\rho_{t} u \cdot \nabla u_{t}\right) \cdot u_{t} d x \\
\leq & C\left(\left\|\rho_{t t}\right\|_{L^{2}}\|u\|_{L^{\infty}}\|\nabla u\|_{L^{4}}\left\|u_{t}\right\|_{L^{4}}+\left\|\rho_{t}\right\|_{L^{4}}\|\nabla u\|_{L^{4}}\left\|u_{t}\right\|_{L^{4}}^{2}\right. \\
& \left.+\left\|\rho_{t}\right\|_{L^{4}}\|u\|_{L^{\infty}}\left\|\nabla u_{t}\right\|_{L^{2}}\left\|u_{t}\right\|_{L^{4}}\right) \\
\leq & C\left(\left\|\rho_{t t}\right\|_{L^{2}}^{2}+\left\|\nabla u_{t}\right\|_{L^{2}}^{2}\right) \leq C\left(1+\left\|\nabla u_{t}\right\|_{L^{2}}^{2}\right),
\end{aligned}
$$




$$
\begin{gathered}
L_{3}+L_{4}=-\int \rho u_{t} \cdot \nabla u \cdot u_{t t} d x-\int \rho u \cdot \nabla u_{t} \cdot u_{t t} d x \\
\leq\left\|\sqrt{\rho} u_{t t}\right\|_{L^{2}}\left\|u_{t}\right\|_{L^{4}}\|\nabla u\|_{L^{4}}+\left\|\sqrt{\rho} u_{t t}\right\|_{L^{2}}\|u\|_{L^{\infty}}\left\|\nabla u_{t}\right\|_{L^{2}} \\
\leq \frac{1}{4}\left\|\sqrt{\rho} u_{t t}\right\|_{L^{2}}^{2}+C\left\|\nabla u_{t}\right\|_{L^{2}}^{2}, \\
L_{5}=-\int P_{t t} \operatorname{div} u_{t} d x \leq\left\|P_{t t}\right\|_{L^{2}}^{2}+\left\|\nabla u_{t}\right\|_{L^{2}}^{2} \leq C+\left\|\nabla u_{t}\right\|_{L^{2}}^{2}, \\
L_{6}+L_{7}+L_{8}=-\int \rho_{t t} \theta e_{2} \cdot u_{t} d x-\int \rho_{t} \theta_{t} e_{2} \cdot u_{t} d x+\int \rho \theta_{t} e_{2} \cdot u_{t t} d x \\
\leq\left\|\rho_{t t}\right\|_{L^{2}}\|\theta\|_{L^{\infty}}\left\|u_{t}\right\|_{L^{2}}+\left\|\rho_{t}\right\|_{L^{4}}\left\|\theta_{t}\right\|_{L^{2}}\left\|u_{t}\right\|_{L^{4}}+\left\|\sqrt{\rho} u_{t t}\right\|_{L^{2}}\left\|\theta_{t}\right\|_{L^{2}} \\
\leq \frac{1}{4}\left\|\sqrt{\rho} u_{t t}\right\|_{L^{2}}^{2}+\left(\left\|\rho_{t t}\right\|_{L^{2}}^{2}+\left\|\nabla u_{t}\right\|_{L^{2}}^{2}+\left\|\theta_{t}\right\|_{L^{2}}^{2}\right) .
\end{gathered}
$$

At last, integrating (4.29) over time (0,T) and inserting estimates (4.30)-(4.35), we have

$$
\int \mu\left|\nabla u_{t}\right|^{2} d x+\int_{0}^{T} \int \rho u_{t t}^{2} d x d s \leq C+\int_{0}^{T} C\left(1+\left\|\nabla u_{t}\right\|_{L^{2}}^{2}\right)\left\|\nabla u_{t}\right\|_{L^{2}}^{2} d s,
$$

from which, together with Gronwall's inequality, (4.22) is obtained immediately. This completes the proof of Lemma 4.3.

Lemma 4.4 Let $(\rho, u, \theta)$ be a classical solution of $(1.2),(1.6)-(1.7)$ on $\Omega \times(0, T]$, under the condition of Theorem 1.1, the following estimates hold:

$$
\begin{aligned}
& \sup _{0 \leq t \leq T}\left(\|\rho\|_{H^{3}}+\|P\|_{H^{3}}\right) \leq C(T), \\
& \sup _{0 \leq t \leq T}\|\nabla u\|_{H^{2}}^{2}+\int_{0}^{T}\left(\left\|\nabla u_{t}\right\|_{H^{1}}^{2}+\|\nabla u\|_{H^{3}}^{2}\right) d s \leq C(T) . \\
& \sup _{0 \leq t \leq T}\left(\left\|\nabla \theta_{t}\right\|_{L^{2}}^{2}+\left\|\nabla^{3} \theta\right\|_{L^{2}}^{2}\right)+\int_{0}^{T}\left(\left\|\nabla^{4} \theta\right\|_{L^{2}}^{2}+\left\|\theta_{t t}\right\|_{L^{2}}^{2}\right) d s \leq C(T) .
\end{aligned}
$$

Proof It follows from Lemma 4.3 that

$$
\begin{aligned}
\|\nabla(\rho \dot{u})\|_{L^{2}} \leq & \left\|\nabla \rho u_{t}\right\|_{L^{2}}+\left\|\rho \nabla u_{t}\right\|_{L^{2}}+\|\nabla \rho u \cdot \nabla u\|_{L^{2}}+\|\rho \nabla u \cdot \nabla u\|_{L^{2}}+\left\|\rho u \cdot \nabla^{2} u\right\|_{L^{2}} \\
\leq & \|\nabla \rho\|_{L^{4}}\left\|u_{t}\right\|_{L^{4}}+\left\|\nabla u_{t}\right\|_{L^{2}}+\|\nabla \rho\|_{L^{4}}\|u\|_{L^{\infty}}\|\nabla u\|_{L^{4}} \\
& +\|\nabla u\|_{L^{4}}^{2}+\|u\|_{L^{\infty}}\left\|\nabla^{2} u\right\|_{L^{2}}^{2} \\
\leq & C
\end{aligned}
$$

which together with Lemma 4.1 gives

$$
\sup _{0 \leq t \leq T}\|\rho \dot{u}\|_{H^{1}} \leq C
$$

The standard $H^{1}$ estimate for elliptic system (3.17) yields

$$
\left\|\nabla^{2} u\right\|_{H^{1}} \leq C\left(\|\rho \dot{u}\|_{H^{1}}+\|\nabla P\|_{H^{1}}+\left\|\rho \theta e_{2}\right\|_{H^{1}}\right) \leq C .
$$


Then, as a consequence of (4.2) and (4.42), we have

$$
\sup _{0 \leq t \leq T}\|\nabla u\|_{H^{2}} \leq C
$$

Moreover, the standard $L^{2}$-estimate for elliptic system (3.17) and Lemma 4.3 yields that

$$
\begin{aligned}
\left\|\nabla^{2} u_{t}\right\|_{L^{2}} \leq & C\left(\left\|\rho u_{t t}\right\|_{L^{2}}+\left\|\rho_{t} u_{t}\right\|_{L^{2}}+\left\|\rho_{t} u \cdot \nabla u\right\|_{L^{2}}+\left\|\rho u_{t} \cdot \nabla u\right\|_{L^{2}}+\left\|\rho u \cdot \nabla u_{t}\right\|_{L^{2}}\right. \\
& \left.+\left\|\nabla P_{t}\right\|_{L^{2}}+\left\|\rho_{t} \theta e_{2}\right\|_{L^{2}}+\left\|\rho \theta_{t} e_{2}\right\|_{L^{2}}\right) \\
\leq & C\left(\left\|\rho u_{t t}\right\|_{L^{2}}+\left\|\rho_{t}\right\|_{L^{4}}\left\|u_{t}\right\|_{L^{4}}+\left\|\rho_{t}\right\|_{L^{4}}\|u\|_{L^{\infty}}\|\nabla u\|_{L^{4}}+\left\|u_{t}\right\|_{L^{4}}\|\nabla u\|_{L^{4}}\right. \\
& \left.+\|u\|_{L^{\infty}}\left\|\nabla u_{t}\right\|_{L^{2}}+\left\|\nabla P_{t}\right\|_{L^{2}}+\left\|\rho_{t}\right\|_{L^{4}}\|\theta\|_{L^{4}}+\left\|\theta_{t}\right\|_{L^{2}}\right) \\
\leq & C\left(\left\|\rho u_{t t}\right\|_{L^{2}}+\left\|\theta_{t}\right\|_{L^{2}}+1\right),
\end{aligned}
$$

which together with (4.22) implies

$$
\int_{0}^{T}\left\|\nabla u_{t}\right\|_{H^{1}}^{2} d s \leq C
$$

On the other hand, applying the standard $H^{2}$-estimate for elliptic system (3.17) again leads to

$$
\begin{aligned}
\left\|\nabla^{2} u\right\|_{H^{2}} & \leq C\left(\|\rho \dot{u}\|_{H^{2}}+\|\nabla P\|_{H^{2}}+\left\|\rho \theta e_{2}\right\|_{H^{2}}\right) \\
& \leq C\left(\left\|\nabla^{2}\left(\rho u_{t}\right)\right\|_{L^{2}}+\left\|\nabla^{2}(\rho u \cdot \nabla u)\right\|_{L^{2}}+\left\|\nabla^{3} P\right\|_{L^{2}}+1\right),
\end{aligned}
$$

where

$$
\begin{aligned}
\left\|\nabla^{2}\left(\rho u_{t}\right)\right\|_{L^{2}} & \leq C\left(\left\|\nabla^{2} \rho u_{t}\right\|_{L^{2}}+\left\|\nabla \rho \nabla u_{t}\right\|_{L^{2}}+\left\|\nabla^{2} u_{t}\right\|_{L^{2}}\right) \\
& \leq C\left(\left\|\nabla^{2} \rho\right\|_{L^{2}}\left\|u_{t}\right\|_{L^{\infty}}+\|\nabla \rho\|_{L^{4}}\left\|\nabla u_{t}\right\|_{L^{4}}+\left\|\nabla^{2} u_{t}\right\|_{L^{2}}\right) \\
& \leq C\left(\left\|\nabla^{2} \rho\right\|_{L^{2}}\left\|\nabla u_{t}\right\|_{H^{1}}+\|\nabla \rho\|_{H^{1}}\left\|\nabla u_{t}\right\|_{H^{1}}+\left\|\nabla^{2} u_{t}\right\|_{L^{2}}\right) \\
& \leq C\left\|\nabla u_{t}\right\|_{H^{1}},
\end{aligned}
$$

and

$$
\begin{aligned}
\left\|\nabla^{2}(\rho u \cdot \nabla u)\right\|_{L^{2}} \leq & C\left(\left\|\nabla^{2} \rho u \cdot \nabla u\right\|_{L^{2}}+\left\|\nabla^{2} u \cdot \nabla u\right\|_{L^{2}}+\left\|u \cdot \nabla^{3} u\right\|_{L^{2}}\right. \\
& \left.+\|\nabla \rho \nabla u \cdot \nabla u\|_{L^{2}}+\left\|\nabla \rho u \cdot \nabla^{2} u\right\|_{L^{2}}\right) \\
\leq & C\left(\left\|\nabla^{2} \rho u \cdot \nabla u\right\|_{L^{2}}+\left\|\nabla^{2} u \cdot \nabla u\right\|_{L^{2}}+\left\|u \cdot \nabla^{3} u\right\|_{L^{2}}\right. \\
& \left.+\|\nabla \rho \nabla u \cdot \nabla u\|_{L^{2}}+\left\|\nabla \rho u \cdot \nabla^{2} u\right\|_{L^{2}}\right) \\
\leq & C\left(\left\|\nabla^{2} \rho\right\|_{L^{2}}\|u\|_{L^{\infty}}\|\nabla u\|_{L^{\infty}}+\left\|\nabla^{2}\right\|_{L^{4}}\|\nabla u\|_{L^{4}}+\|u\|_{L^{\infty}}\left\|\nabla^{3} u\right\|_{L^{2}}\right. \\
& \left.+\|\nabla \rho\|_{L^{6}}\|\nabla u\|_{L^{6}}^{2}+\|\nabla \rho\|_{L^{4}}\|u\|_{L^{\infty}}\left\|\nabla^{2} u\right\|_{L^{4}}\right) \\
\leq & C .
\end{aligned}
$$


In order to estimate the third term on the right-hand side of (4.46), applying $\nabla^{3}$ to (4.18) and integrating the resulting equation over $\Omega$, we obtain

$$
\begin{aligned}
\frac{d}{d t} \| & \nabla^{3} P \|_{L^{2}}^{2} \\
\leq & C\left(\left\|\nabla^{3} u \cdot \nabla P\right\|_{L^{2}}+\left\|\nabla u \cdot \nabla^{3} P\right\|_{L^{2}}+\left\|\nabla^{2} u \cdot \nabla^{2} P\right\|_{L^{2}}+\left\|\nabla^{4} u P\right\|_{L^{2}}\right) \\
\leq & C\left(\left\|\nabla^{3} u\right\|_{L^{2}}\|\nabla P\|_{L^{\infty}}+\|\nabla u\|_{L^{\infty}}\left\|\nabla^{3} P\right\|_{L^{2}}+\left\|\nabla^{2} u\right\|_{L^{4}}\left\|\nabla^{2} P\right\|_{L^{4}}+\left\|\nabla^{4} u\right\|_{L^{2}}\right) \\
\leq & C\left(\left\|\nabla^{3} P\right\|_{L^{2}}+\left\|\nabla^{4} u\right\|_{L^{2}}\right) \\
\leq & C\left(\left\|\nabla^{3} P\right\|_{L^{2}}+\left\|\nabla^{2}(\rho \dot{u})\right\|_{L^{2}}+\left\|\nabla^{2}\left(\rho \theta e_{2}\right)\right\|_{L^{2}}\right) \\
\leq & C\left(\left\|\nabla^{3} P\right\|_{L^{2}}+\left\|\nabla^{2}\left(\rho u_{t}\right)\right\|_{L^{2}}+\left\|\nabla^{2}(u \cdot \nabla u)\right\|_{L^{2}}+1\right) \\
\leq & C\left(\left\|\nabla^{3} P\right\|_{L^{2}}+\left\|\nabla^{2} \rho\right\|_{L^{2}}\left\|u_{t}\right\|_{L^{\infty}}+\left\|\nabla^{2} u_{t}\right\|_{L^{2}}+\|\nabla \rho\|_{L^{4}}\left\|\nabla u_{t}\right\|_{L^{4}}\right. \\
& \left.+\left\|\nabla^{2} u \cdot \nabla u\right\|_{L^{2}}+\left\|u \cdot \nabla^{3} u\right\|_{L^{2}}+\left\|\nabla u \cdot \nabla^{2} u\right\|_{L^{2}}+1\right) \\
\leq & C\left(\left\|\nabla^{3} P\right\|_{L^{2}}+\left\|\nabla u_{t}\right\|_{H^{1}}+\|\nabla \rho\|_{H^{1}}\left\|\nabla u_{t}\right\|_{H^{1}}+\left\|\nabla^{2} u\right\|_{L^{4}}\|\nabla u\|_{L^{4}}\right. \\
& \left.+\|u\|_{L^{\infty}}\left\|\nabla^{3} u\right\|_{L^{2}}+\|\nabla u\|_{L^{\infty}}\left\|\nabla^{2} u\right\|_{L^{2}}+1\right) \\
\leq & C\left(\left\|\nabla^{3} P\right\|_{L^{2}}+\left\|\nabla u_{t}\right\|_{H^{1}}+1\right),
\end{aligned}
$$

which, together with Gronwall's inequality (4.45), implies that

$$
\sup _{0 \leq t \leq T}\left\|\nabla^{3} P\right\|_{L^{2}} \leq C
$$

Taking (4.45)-(4.50) into consideration, we have

$$
\int_{0}^{T}\|\nabla u\|_{H^{3}}^{2} d s \leq C
$$

It is easy to check that similar arguments work for $\rho$ by using (4.51).

At last, in order to estimate the third-order derivative of temperature, differentiating $(1.2)_{3}$ with respect to $t$, we get

$$
\theta_{t t}+u_{t} \cdot \nabla \theta-u \cdot \nabla \theta_{t}-\kappa \Delta \theta_{t}=0
$$

Then, multiplying (4.52) by $\theta_{t t}$ and then integrating the resulting equation over $\Omega$, after integration by parts, we obtain

$$
\begin{aligned}
\frac{\kappa}{2} \frac{d}{d t}\left\|\nabla \theta_{t}\right\|_{L^{2}}^{2}+\left\|\theta_{t t}\right\|_{L^{2}}^{2} & =-\int u_{t} \cdot \nabla \theta \theta_{t t} d x-\int u \cdot \nabla \theta_{t} \theta_{t t} d x \\
& \leq \frac{1}{2}\left\|\theta_{t t}\right\|_{L^{2}}^{2}+\int\left|u_{t} \cdot \nabla \theta\right|^{2} d x+\int\left|u \cdot \nabla \theta_{t}\right|^{2} d x \\
& \leq \frac{1}{2}\left\|\theta_{t t}\right\|_{L^{2}}^{2}+\left\|u_{t}\right\|_{L^{4}}^{2}\|\nabla \theta\|_{L^{4}}^{2}+\|u\|_{L^{\infty}}^{2}\left\|\nabla \theta_{t}\right\|_{L^{2}}^{2} \\
& \leq \frac{1}{2}\left\|\theta_{t t}\right\|_{L^{2}}^{2}+C\left(\left\|\nabla u_{t}\right\|_{L^{2}}^{2}\|\nabla \theta\|_{H^{1}}^{2}+\|u\|_{H^{2}}^{2}\left\|\nabla \theta_{t}\right\|_{L^{2}}^{2}\right) \\
& \leq \frac{1}{2}\left\|\theta_{t t}\right\|_{L^{2}}^{2}+C\left(1+\|u\|_{H^{2}}^{2}\left\|\nabla \theta_{t}\right\|_{L^{2}}^{2}\right),
\end{aligned}
$$


from which, together with Gronwall's inequality, we have

$$
\sup _{0 \leq t \leq T}\left\|\nabla \theta_{t}\right\|_{L^{2}}^{2}+\int_{0}^{T}\left\|\theta_{t t}\right\|_{L^{2}}^{2} d s \leq C(T) .
$$

Now, we apply the operator $\nabla^{3}$ on both sides of equation $(1.2)_{3}$, then we have

$$
\begin{aligned}
& \frac{1}{2} \frac{d}{d t}\left\|\nabla^{3} \theta\right\|_{L^{2}}^{2}+\kappa\left\|\nabla^{4} \theta\right\|_{L^{2}}^{2} \\
&=-\int \nabla^{3}(u \cdot \nabla) \theta \nabla^{3} \theta d x \\
&=-\int\left(\nabla^{3} u \cdot \nabla\right) \theta \nabla^{3} \theta d x-\int\left(u \cdot \nabla^{4}\right) \theta \nabla^{3} \theta d x-3 \int\left(\nabla^{2} u \cdot \nabla^{2}\right) \theta \nabla^{3} \theta d x \\
& \quad-3 \int\left(\nabla u \cdot \nabla^{3}\right) \theta \nabla^{3} \theta d x \\
& \leq C\left(\left\|\nabla^{3} u\right\|_{L^{2}}\|\nabla \theta\|_{L^{4}}\left\|\nabla^{3} \theta\right\|_{L^{4}}+\|u\|_{L^{\infty}}\left\|\nabla^{4} \theta\right\|_{L^{2}}\left\|\nabla^{3} \theta\right\|_{L^{2}}+\left\|\nabla^{3} u\right\|_{L^{2}}\left\|\nabla^{2} \theta\right\|_{L^{4}}^{2}\right. \\
&\left.\quad+\|\nabla u\|_{L^{\infty}}\left\|\nabla^{3} \theta\right\|_{L^{2}}^{2}\right) \\
& \leq C\left(\left\|\nabla^{3} \theta\right\|_{L^{2}}^{\frac{1}{2}}\left\|\nabla^{4} \theta\right\|_{L^{2}}^{\frac{1}{2}}+\left\|\nabla^{4} \theta\right\|_{L^{2}}\left\|\nabla^{3} \theta\right\|_{L^{2}}+\left\|\nabla^{3} \theta\right\|_{L^{2}}\left\|\nabla^{2} \theta\right\|_{L^{2}}+\left\|\nabla^{3} \theta\right\|_{L^{2}}^{2}\right) \\
& \leq \frac{\kappa}{2}\left\|\nabla^{4} \theta\right\|_{L^{2}}^{2}+C\left(1+\left\|\nabla^{3} \theta\right\|_{L^{2}}^{2}\right),
\end{aligned}
$$

from which, after integration over $(0, T),(4.39)$ is proved. Hence, the proof of Lemma 4.4 is completed.

Lemma 4.5 Let $(\rho, u, \theta)$ be a classical solution of (1.2), (1.6)-(1.7) on $\Omega \times(0, T]$, under the condition of Theorem 1.1, the following estimates hold:

$$
\begin{aligned}
& \sup _{0 \leq t \leq T}\left(\left\|\nabla u_{t}\right\|_{H^{1}}^{2}+\left\|\nabla^{4} u\right\|_{L^{2}}^{2}\right)+\int_{0}^{T}\left\|\nabla u_{t t}\right\|_{L^{2}}^{2} d s \leq C(T), \\
& \sup _{0 \leq t \leq T}\left(\left\|\nabla \theta_{t}\right\|_{H^{1}}^{2}+\left\|\nabla^{4} \theta\right\|_{L^{2}}^{2}\right)+\int_{0}^{T}\left\|\nabla \theta_{t t}\right\|_{L^{2}}^{2} d s \leq C(T) .
\end{aligned}
$$

Proof First, differentiating $(1.2)_{2}$ with respect to $t$ twice, one can get

$$
\begin{aligned}
\rho u_{t t t} & +\rho u \cdot \nabla u_{t t}-\mu \Delta u_{t t} \\
= & 2 \operatorname{div}(\rho u) u_{t t}+\operatorname{div}(\rho u)_{t} u_{t}-2(\rho u)_{t} \cdot \nabla u_{t}-\left(\rho_{t t} u+2 \rho_{t} u_{t}\right) \cdot \nabla u \\
& -\rho u_{t t} \cdot \nabla u-\nabla P_{t t}+\rho_{t t} \theta e_{2}+2 \rho_{t} \theta_{t} e_{2}+\rho \theta_{t t} e_{2} .
\end{aligned}
$$

Multiplying (4.58) by $u_{t t}$ and then integrating the resulting equation over $\Omega$, after integration by parts, we obtain

$$
\begin{aligned}
& \frac{1}{2} \frac{d}{d t} \int \rho\left|u_{t t}\right|^{2} d x+\int \mu\left|\nabla u_{t t}\right|^{2} d x \\
& \quad=-4 \int \rho u u_{t t} \cdot \nabla u_{t t} d x-\int(\rho u)_{t} \cdot\left(\nabla\left(u_{t} \cdot u_{t t}\right)+2 \nabla u_{t} \cdot u_{t t}\right) d x
\end{aligned}
$$




$$
\begin{aligned}
& \quad-\int\left(\rho_{t t} u+2 \rho_{t} u_{t}\right) \cdot \nabla u \cdot u_{t t} d x-\int \rho u_{t t} \cdot \nabla u \cdot u_{t t} d x+\int P_{t t} \operatorname{div} u_{t t} d x \\
& \quad+\int\left(\rho_{t t} \theta e_{2}+2 \rho_{t} \theta_{t} e_{2}+\rho \theta_{t t} e_{2}\right) d x \\
& :=\sum_{i=1}^{6} M_{i} .
\end{aligned}
$$

Next, we estimate each term $M_{i}, i=1,2,3,4,5$, as follows:

$$
\begin{aligned}
M_{1} & =-4 \int \rho u u_{t t} \cdot \nabla u_{t t} d x \leq C\|u\|_{L^{\infty}}\left\|\sqrt{\rho} u_{t t}\right\|_{L^{2}}\left\|\nabla u_{t t}\right\|_{L^{2}} \\
& \leq \delta\left\|\nabla u_{t t}\right\|_{L^{2}}^{2}+C\left\|\sqrt{\rho} u_{t t}\right\|_{L^{2}}^{2} .
\end{aligned}
$$

It follows from Lemma 4.2, Lemma 4.3, and Lemma 4.4 that

$$
\begin{aligned}
& M_{2}=-\int(\rho u)_{t} \cdot\left(\nabla\left(u_{t} \cdot u_{t t}\right)+2 \nabla u_{t} \cdot u_{t t}\right) d x \\
& \leq C\left(\left\|\rho_{t} u\right\|_{L^{4}}+\left\|\rho u_{t}\right\|_{L^{4}}\right)\left(\left\|\nabla u_{t}\right\|_{L^{2}}\left\|u_{t t}\right\|_{L^{4}}+\left\|u_{t}\right\|_{L^{4}}\left\|\nabla u_{t t}\right\|_{L^{2}}\right) \\
& \leq C\left(\left\|\rho_{t}\right\|_{H^{1}}+\left\|u_{t}\right\|_{H^{1}}\right)\left(\left\|u_{t t}\right\|_{L^{2}}^{\frac{1}{2}}\left\|\nabla u_{t t}\right\|_{L^{2}}^{\frac{1}{2}}+\left\|u_{t}\right\|_{H^{1}}\left\|\nabla u_{t t}\right\|_{L^{2}}\right) \\
& \leq \delta\left\|\nabla u_{t t}\right\|_{L^{2}}^{2}+C \\
& M_{3}=-\int\left(\rho_{t t} u+2 \rho_{t} u_{t}\right) \cdot \nabla u \cdot u_{t t} d x \\
& \leq C\left(\left\|\rho_{t t}\right\|_{L^{2}}\|u\|_{L^{\infty}}+\left\|\rho_{t}\right\|_{L^{4}}\left\|u_{t}\right\|_{L^{4}}\right)\|\nabla u\|_{L^{4}}\left\|u_{t t}\right\|_{L^{4}} \\
& \leq C\left(\left\|\rho_{t t}\right\|_{L^{2}}\|u\|_{H^{2}}+\left\|\rho_{t}\right\|_{H^{1}}\left\|u_{t}\right\|_{L^{2}}^{\frac{1}{2}}\left\|\nabla u_{t}\right\|_{L^{2}}^{\frac{1}{2}}\right)\|\nabla u\|_{H^{1}}\left\|u_{t t}\right\|_{L^{2}}^{\frac{1}{2}}\left\|\nabla u_{t t}\right\|_{L^{2}}^{\frac{1}{2}} \\
& \leq \delta\left\|\nabla u_{t t}\right\|_{L^{2}}^{2}+C\left\|\rho_{t t}\right\|_{L^{2}}^{2}, \\
& M_{4}+M_{5}=-\int \rho u_{t t} \cdot \nabla u \cdot u_{t t} d x+\int P_{t t} \operatorname{div} u_{t t} d x \\
& \quad \leq C\left(\left\|\sqrt{\rho} u_{t t}\right\|_{L^{2}}\|\nabla u\|_{L^{4}}\left\|u_{t t}\right\|_{L^{4}}+\left\|P_{t t}\right\|_{L^{2}}\left\|\nabla u_{t t}\right\|_{L^{2}}\right) \\
& \quad \leq C\left(\left\|\sqrt{\rho} u_{t t}\right\|_{L^{2}}\|\nabla u\|_{H^{1}}\left\|u_{t t}\right\|_{L^{2}}^{\frac{1}{2}}\left\|\nabla u_{t t}\right\|_{L^{2}}^{\frac{1}{2}}+\left\|P_{t t}\right\|_{L^{2}}\left\|\nabla u_{t t}\right\|_{L^{2}}\right) \\
& \quad \leq \delta\left\|\nabla u_{t t}\right\|_{L^{2}}^{2}+C\left(\left\|\sqrt{\rho} u_{t t}\right\|_{L^{2}}^{2}+\left\|P_{t t}\right\|_{L^{2}}^{2}\right) \\
& M_{6}=\int\left(\rho_{t t} \theta e_{2}+2 \rho_{t} \theta \theta_{t} e_{2}+\rho \theta_{t t} e_{2}\right) d x \\
& \leq C\left(\left\|\rho_{t t}\right\|_{L^{2}}\|\theta\|_{L^{\infty}}+\left\|\rho_{t}\right\|_{L^{2}}\left\|\theta_{t}\right\|_{L^{2}}+\|\rho\|_{L^{\infty}}\left\|\theta_{t t}\right\|_{L^{2}}\right) \\
& \leq C\left(\left\|\rho_{t t}\right\|_{L^{2}}+\left\|\theta_{t t}\right\|_{L^{2}}+1\right) .
\end{aligned}
$$

Substituting (4.60)-(4.64) into (4.59) and choosing $\delta$ suitably small, we get

$$
\begin{aligned}
& \frac{d}{d t} \int \rho\left|u_{t t}\right|^{2} d x+\int \mu\left|\nabla u_{t t}\right|^{2} d x \\
& \quad \leq C\left(\left\|\sqrt{\rho} u_{t t}\right\|_{L^{2}}^{2}+\left\|\rho_{t t}\right\|_{L^{2}}^{2}+\left\|P_{t t}\right\|_{L^{2}}^{2}+\left\|\theta_{t t}\right\|_{L^{2}}+1\right) .
\end{aligned}
$$


Then, integrating inequality (4.65) over $(0, T)$, together with (4.21) and Gronwall's inequality, yields that

$$
\sup _{0 \leq t \leq T} \int \rho\left|u_{t t}\right|^{2} d x+\int_{0}^{T} \int\left|\nabla u_{t t}\right|^{2} d x d s \leq C(T) .
$$

Then (4.56) follows from (4.22) and (4.44).

Next, differentiating $(1.2)_{3}$ with respect to $t$ twice, we get

$$
\theta_{t t t}-\kappa \Delta \theta_{t t}=-u_{t t} \cdot \nabla \theta-2 u_{t} \cdot \nabla \theta_{t}-u \cdot \nabla \theta_{t t}
$$

Multiplying (4.67) by $\theta_{t t}$ and then integrating the resulting equation over $\Omega$, after integration by parts, we have

$$
\begin{aligned}
\frac{1}{2} \frac{d}{d t} & \left\|\theta_{t t}\right\|_{L^{2}}^{2}+\kappa\left\|\nabla \theta_{t t}\right\|_{L^{2}}^{2} \\
= & -\int[(u \cdot \nabla) \theta]_{t t} \theta_{t t} d x \\
= & -\int\left(u_{t t} \cdot \nabla \theta+2 u_{t} \cdot \nabla \theta_{t}+u \cdot \nabla \theta_{t t}\right) \theta_{t t} d x \\
\leq & C\left(\left\|u_{t t}\right\|_{L^{4}}\|\nabla \theta\|_{L^{2}}\left\|\theta_{t t}\right\|_{L^{4}}+\left\|u_{t}\right\|_{L^{4}}\left\|\nabla \theta_{t}\right\|_{L^{2}}\left\|\theta_{t t}\right\|_{L^{4}}+\|\nabla u\|_{L^{2}}\left\|\theta_{t t}\right\|_{L^{4}}^{2}\right) \\
\leq & C\left(\left\|u_{t t}\right\|_{L^{2}}^{\frac{1}{2}}\left\|\nabla u_{t t}\right\|_{L^{2}}^{\frac{1}{2}}\left\|\theta_{t t}\right\|_{L^{2}}^{\frac{1}{2}}\left\|\nabla \theta_{t t}\right\|_{L^{2}}^{\frac{1}{2}}+\left\|u_{t}\right\|_{L^{2}}^{\frac{1}{2}}\left\|\nabla u_{t}\right\|_{L^{2}}^{\frac{1}{2}}\left\|\nabla \theta_{t}\right\|_{L^{2}}\left\|\theta_{t t}\right\|_{L^{2}}^{\frac{1}{2}}\left\|\nabla \theta_{t t}\right\|_{L^{2}}^{\frac{1}{2}}\right. \\
& \left.+\left\|\theta_{t t}\right\|_{L^{2}}\left\|\nabla \theta_{t t}\right\|_{L^{2}}\right) \\
\leq & C\left(\left\|\nabla u_{t t}\right\|_{L^{2}}\left\|\nabla \theta_{t t}\right\|_{L^{2}}+\left\|\nabla u_{t}\right\|_{L^{2}}\left\|\nabla \theta_{t}\right\|_{L^{2}}\left\|\nabla \theta_{t t}\right\|_{L^{2}}+\left\|\theta_{t t}\right\|_{L^{2}}\left\|\nabla \theta_{t t}\right\|_{L^{2}}\right) \\
\leq & \frac{\kappa}{2}\left\|\nabla \theta_{t t}\right\|_{L^{2}}^{2}+C\left(1+\left\|\nabla u_{t t}\right\|_{L^{2}}^{2}+\left\|\theta_{t t}\right\|_{L^{2}}\right),
\end{aligned}
$$

where in the last inequality we have used (4.22) and (4.39). Then, integrating inequality (4.68) over $(0, T)$, together with (4.56) and Gronwall's inequality, yields that

$$
\sup _{0 \leq t \leq T}\left\|\theta_{t t}\right\|_{L^{2}}^{2}+\int_{0}^{T} \kappa\left\|\nabla \theta_{t t}\right\|_{L^{2}}^{2} d s \leq C(T) .
$$

Furthermore, the standard $L^{2}$-estimate for elliptic system (4.52) and Lemma 4.3 yields that

$$
\begin{aligned}
\left\|\nabla^{2} \theta_{t}\right\|_{L^{2}} & \leq C\left(\left\|\theta_{t t}\right\|_{L^{2}}+\left\|u_{t} \cdot \nabla \theta\right\|_{L^{2}}+\left\|u \cdot \nabla \theta_{t}\right\|_{L^{2}}\right) \\
& \leq C\left(1+\left\|u_{t}\right\|_{L^{4}}\|\nabla \theta\|_{L^{4}}+\|u\|_{L^{\infty}}\left\|\nabla \theta_{t}\right\|_{L^{2}}\right) \\
& \leq C\left(1+\left\|\nabla u_{t}\right\|_{L^{2}}\left\|\nabla^{2} \theta\right\|_{L^{2}}+\left\|\nabla \theta_{t}\right\|_{L^{2}}\right) \\
& \leq C
\end{aligned}
$$

where we have used (4.22), (4.39), (4.69). Hence, combining (1.2) 3 , (4.70), and Lemma 4.4, by using elliptic estimate, we have

$$
\left\|\nabla^{4} \theta\right\|_{L^{2}} \leq C\left(\left\|\nabla^{2} \theta_{t}\right\|_{L^{2}}+\left\|\nabla u \cdot \nabla^{2} \theta\right\|_{L^{2}}+\left\|u \cdot \nabla^{3} \theta\right\|_{H^{2}}\right) \leq C,
$$

by which we finish the proof of Lemma 4.5. 
Finally, by using the continuity argument, we can extend the local classical solution to a global one, and thus Theorem 1.1 is proved.

\begin{abstract}
Acknowledgements
The authors sincerely appreciate the anonymous referees for their careful reading and suggestions for the paper. The authors would also like to thank Journal of Inequalities and Applications for considering this paper to be published.
\end{abstract}

\title{
Funding
}

The second author's research was supported in part by the Chinese National Natural Science Foundation under grant 11571232 and 11831011.

\section{Availability of data and materials}

Not applicable.

\section{Competing interests}

The authors declare that they have no competing interests.

\section{Authors' contributions}

The authors have made the same contribution. All authors read and approved the final manuscript.

\section{Author details}

${ }^{1}$ School of Finance, Shanghai Lixin University of Accounting and Finance, Shanghai 201209, P.R. China. ${ }^{2}$ School of Mathematical Sciences, Shanghai Jiao Tong University, Shanghai 200240, P.R. China. ${ }^{3}$ School of Statistics and Mathematics, Shanghai Lixin University of Accounting and Finance, Shanghai, 201209, P.R. China.

\section{Publisher's Note}

Springer Nature remains neutral with regard to jurisdictional claims in published maps and institutional affiliations.

Received: 6 June 2020 Accepted: 16 September 2020 Published online: 15 October 2020

\section{References}

1. Abidi, H., Hmidi, T.: On the global well-posedness for Boussinesq system. J. Differ. Equ. 233(1), 199-220 (2007)

2. Abidi, H., Zhang, P.: On the global well-posedness of 2-D Boussinesq system with variable viscosity. Adv. Math. 305, 1202-1249 (2017)

3. Abidi, H., Zhang, P.: On the global well-posedness of 3-D Boussinesq system with variable viscosity. Chin. Ann. Math., Ser. B 40(5), 643-688 (2019)

4. Brandolese, L., Schonbek, M.E.: Large time decay and growth for solutions of a viscous Boussinesq system. Trans. Am. Math. Soc. 364(10), 5057-5090 (2012)

5. Brézis, H., Gallouet, T.: Nonlinear Schrödinger evolution equations. Nonlinear Anal. 4(4), 677-681 (1980)

6. Brézis, H., Wainger, S.: A note on limiting cases of Sobolev embeddings and convolution inequalities. Commun. Partial Differ. Equ. 5(7), 773-789 (1980)

7. Cannon, J.R., DiBenedetto, E.: The initial value problem for the Boussinesq equations with data in $L^{p}$. In: Approximation Methods for Navier-Stokes Problems (Proc. Sympos., Univ. Paderborn, Paderborn, 1979). Lecture Notes in Math., vol. 771, pp. 129-144. Springer, Berlin (1980)

8. Chae, D.: Global regularity for the 2D Boussinesq equations with partial viscosity terms. Adv. Math. 203(2), 497-513 (2006)

9. Chae, D., Kim, S.-K., Nam, H.-S.: Local existence and blow-up criterion of Hölder continuous solutions of the Boussinesq equations. Nagoya Math. J. 155, 55-80 (1999)

10. Chae, D., Nam, H.-S.: Local existence and blow-up criterion for the Boussinesq equations. Proc. R. Soc. Edinb. A 127(5), 935-946 (1997)

11. Cho, Y., Kim, H.: Existence results for viscous polytropic fluids with vacuum. J. Differ. Equ. 228(2), 377-411 (2006)

12. Danchin, R., Paicu, M.: Existence and uniqueness results for the Boussinesq system with data in Lorentz spaces. Physica D 237(10-12), 1444-1460 (2008)

13. Danchin, R., Paicu, M.: Global existence results for the anisotropic Boussinesq system in dimension two. Math. Models Methods Appl. Sci. 21(3), 421-457 (2011)

14. Dong, B.-Q., Ye, Z., Zhai, X.: Global regularity for the 2D Boussinesq equations with temperature-dependent viscosity. J. Math. Fluid Mech. 22(1), Paper No. 2, 16 (2020)

15. Engler, H.: An alternative proof of the Brezis-Wainger inequality. Commun. Partial Differ. Equ. 14(4), 541-544 (1989)

16. Hmidi, T., Rousset, F.: Global well-posedness for the Euler-Boussinesq system with axisymmetric data. J. Funct. Anal. 260(3), 745-796 (2011)

17. Hou, T.Y., Li, C.: Global well-posedness of the viscous Boussinesq equations. Discrete Contin. Dyn. Syst. 12(1), 1-12 (2005)

18. Huang, X., Li, J., Xin, Z.: Global well-posedness of classical solutions with large oscillations and vacuum to the three-dimensional isentropic compressible Navier-Stokes equations. Commun. Pure Appl. Math. 65(4), 549-585 (2012)

19. Jiu, Q., Liu, J.: Global-wellposedness of 2D Boussinesq equations with mixed partial temperature-dependent viscosity and thermal diffusivity. Nonlinear Anal. 132, 227-239 (2016)

20. Li, D., Xu, X.: Global wellposedness of an inviscid 2D Boussinesq system with nonlinear thermal diffusivity. Dyn. Partial Differ. Equ. 10(3), 255-265 (2013) 
21. Li, H., Pan, R., Zhang, W.: Initial boundary value problem for 2D Boussinesq equations with temperature-dependent diffusion. J. Hyperbolic Differ. Equ. 12(3), 469-488 (2015)

22. Li, W., Shang, Z., Tang, F.: Global existence of classical solutions for two-dimensional isentropic compressible Navier-Stokes equations with small initial mass. Adv. Differ. Equ. 2020, 214 (2020)

23. Liu, X., Li, Y.: On the stability of global solutions to the 3D Boussinesq system. Nonlinear Anal. 95, 580-591 (2014)

24. Lorca, S.A., Boldrini, J.L.: The initial value problem for a generalized Boussinesq model. Nonlinear Anal., Theory Methods Appl. 36(4), 457-480 (1999)

25. Majda, A.: Introduction to PDEs and Waves for the Atmosphere and Ocean. Courant Lecture Notes in Mathematics, vol. 9. Am. Math. Soc., Providence (2003)

26. Qin, Y., Wang, Y., Su, X., Zhang, J.: Global existence of solutions for the three-dimensional Boussinesq system with anisotropic data. Discrete Contin. Dyn. Syst. 36(3), 1563-1581 (2016)

27. Qiu, H., Yao, Z:: Well-posedness for density-dependent Boussinesq equations without dissipation terms in Besov spaces. Comput. Math. Appl. 73(9), 1920-1931 (2017)

28. Sawada, O., Taniuchi, Y.: On the Boussinesq flow with nondecaying initial data. Funkc. Ekvacioj 47(2), 225-250 (2004)

29. Shang, Z., Tang, F.: Global existence and exponential decay of strong solutions for the three-dimensional Boussinesq equations. J. Inequal. Appl. 2020, 50, 10 (2020)

30. Su, X., Wang, G., Wang, Y.: Persistence of global well-posedness for the 2D Boussinesq equations with fractional dissipation. Adv. Differ. Equ. 2019, 420, 19 (2019)

31. Sun, Y., Wang, C., Zhang, Z:: A Beale-Kato-Majda blow-up criterion for the 3-D compressible Navier-Stokes equations. J. Math. Pures Appl. (9) 95(1), 36-47 (2011)

32. Sun, Y., Zhang, Z:: Global regularity for the initial-boundary value problem of the 2-D Boussinesq system with variable viscosity and thermal diffusivity. J. Differ. Equ. 255(6), 1069-1085 (2013)

33. Tang, T., Gao, H.: On the compressible Boussinesq equations with partial dissipation term. Taiwan. J. Math. 18(5), 1583-1604 (2014)

34. Wang, C., Zhang, Z.: Global well-posedness for the 2-D Boussinesq system with the temperature-dependent viscosity and thermal diffusivity. Adv. Math. 228(1), 43-62 (2011)

35. Wen, H., Zhu, C.: Global solutions to the three-dimensional full compressible Navier-Stokes equations with vacuum at infinity in some classes of large data. SIAM J. Math. Anal. 49(1), 162-221 (2017)

36. Wen, Z., Ye, Z:: On the global existence of strong solution to the 3D damped Boussinesq equations with zero thermal diffusion. Z. Anal. Anwend. 37(3), 341-348 (2018)

37. $\mathrm{Xu}, \mathrm{X}$.: Local existence and blow-up criterion of the 2-D compressible Boussinesq equations without dissipation terms. Discrete Contin. Dyn. Syst. 25(4), 1333-1347 (2009)

38. Ye, Z: Global regularity for a 3D Boussinesq model without thermal diffusion. Z. Angew. Math. Phys. 68(4), Art. 83, 9 (2017)

39. Ye, Z: On global well-posedness for the 3D Boussinesq equations with fractional partial dissipation. Appl. Math. Lett. 90, 1-7 (2019)

40. Ye, Z: Blow-up criterion of strong solution with vacuum for the $2 \mathrm{D}$ nonhomogeneous density-temperature-dependent Boussinesq equations. Z. Anal. Anwend. 39(1), 83-101 (2020)

41. Ye, Z.: Global regularity results for the $2 \mathrm{D}$ Boussinesq equations and micropolar equations with partial dissipation. J. Differ. Equ. 268(3), 910-944 (2020)

42. Yu, H., Zhao, J.: Global classical solutions to the 3D isentropic compressible Navier-Stokes equations in a bounded domain. Nonlinearity 30(1), 361-381 (2017)

43. Zhai, X., Chen, Z.-M.: Global well-posedness for N-dimensional Boussinesq system with viscosity depending on temperature. Commun. Math. Sci. 16(5), 1427-1449 (2018)

44. Zhai, X., Dong, B.-Q., Chen, Z.-M.: Global well-posedness for 2-D Boussinesq system with the temperature-dependent viscosity and supercritical dissipation. J. Differ. Equ. 267(1), 364-387 (2019)

45. Zhong, X.: Global well-posedness to the Cauchy problem of two-dimensional density-dependent Boussinesa equations with large initial data and vacuum. Discrete Contin. Dyn. Syst. 39(11), 6713-6745 (2019)

46. Zhong, X.: Strong solutions to the 2D Cauchy problem of density-dependent viscous Boussinesq equations with vacuum. J. Math. Phys. 60(5), 051505, 15 (2019)

47. Zlotnik, A.A.: Uniform estimates and the stabilization of symmetric solutions of a system of quasilinear equations. Differ. Uravn. 36(5), 634-646, 718 (2000)

\section{Submit your manuscript to a SpringerOpen ${ }^{\circ}$ journal and benefit from:}

- Convenient online submission

- Rigorous peer review

- Open access: articles freely available online

- High visibility within the field

- Retaining the copyright to your article

Submit your next manuscript at $\gg$ springeropen.com 